\title{
A Study of the Wavelength Calibration of NEWSIPS High-Dispersion Spectra
}

\author{
Myron A. Smith ${ }^{1}$ \\ ${ }^{1}$ STScI/CSC, Space Telescope Science Institute, 3700 San Martin Dr. Baltimore, MD \\ 21218; Email: msmith@stsci.edu
}

Received — 


\begin{abstract}
In this study we cross-correlate many IUE echellograms of a variety of well observed stars to evaluate systematic error sources in the wavelength zero-points (velocities) of all three cameras. We first evaluate differences between the final archived ("NEWSIPS") and the originally processed ("IUESIPS") echellograms. These show a marked time dependence in zero-point for the $S W P$ camera due to several revisions of wavelength calibration coefficients used for IUESIPS. Smaller offsets are present for the $L W R$ camera between the two processings. We also evaluated small-amplitude fluctuations in the zero-points of the NEWSIPS wavelength calibration spectra themselves. In the case of the SWP camera, these variations are too complicated to have been completely removed in the NEWSIPS wavelength calibration. We also examine wavelength zero-point disparities between data obtained both through the small and large entrance apertures as well as for observations made by different target acquisitions of faint and bright stars. We also find that statistical differences between these alternative observing modes are virtually nil. For large-aperture observations the dominant error source is the placement of the target in the aperture. These give rise to non-gaussian, extended "tails" in apparent velocity. We also searched for spurious trends with time. Except for a possible trend for faint objects with $S W P$ camera data, we can not detect significant dependences with time. Additionally, we discovered a trend with telescope focus for datasets derived from intensive monitoring campaigns of bright stars. These exhibit a repeatable, one-day "radial velocity variation" with a semi-amplitude of nearly $3 \mathrm{~km} \mathrm{~s}^{-1}$. This pattern appears to be a by-product of fluctuations in telescope focus caused by operational procedures to maintain the ambient instrument temperature.
\end{abstract}


In the second part of the paper, we measure the mean zero-point errors of NEWSIPS echellogram data with respect to laboratory results by using the Goddard High Resolution Spectrograph (GHRS) spectral atlas of the O9 V spectral standard 10 Lacertae as an intermediary reference. We find that the derived apparent velocity difference for this star is essentially zero: $-1 \pm 3.5$ $\mathrm{km} \mathrm{s}^{-1}$. Several less precise comparisons lead to similar results, including cross-correlations of: (1) spectra of 10 Lac and two stars with similar spectra, HD 93521 and HD 60753, (2) lines in common with the SWP camera and GHRS and STIS atlases of Arcturus and Procyon, and (3) interstellar lines in the GHRS spectrum of the white dwarf G191-B2B. The zero-points of the NEWSIPS-processed long-wavelength cameras are evaluated and are also found to be nearly zero $\left( \pm 5 \mathrm{~km} \mathrm{~s}^{-1}\right)$ relative to the Arcturus and Procyon atlas calibrations and relative to one another. In general, these results do not support the suggestion by González-Riestra that corrections should be introduced to the wavelength scales of various NEWSIPS high-dispersion data products. Despite our optimistic results, it is obvious that using small $I U E$ datasets from large-aperture observations of arbitrarily chosen stars can contain velocity errors of at least a few $\mathrm{km} \mathrm{s}^{-1}$. 


\section{Introduction}

During its operational lifetime (26 January 1978 to 30 September 1996) the International Ultraviolet Explorer satellite observed over 3700 objects outside the Solar System in its high dispersion mode. Because a high percentage of these objects have been regularly observed by other satellites and from the ground, a comparison of radial velocities obtained from this instrument is of considerable value to multi-wavelength and time-monitoring investigations that utilize $I U E$ data. However stable a spectrograph might be, its calibration is susceptible to systematic wavelengths errors, usually arising from such real-world complications as the differing paths of the stellar and emission-line calibration beams through the spectrograph, dispersion nonlinearities, and in the case of the IUE, both image placement in the aperture and a wandering of its echelle spectral format on the detector surface. In this paper one of our goals will be to examine several potential sources of systematic error as a function of various instrumental parameters by means of standard cross-correlation tools. Our purpose is to give a general evaluation of the robustness of the wavelength calibration of the "NEWSIPS' ' which, in contrast to its predecessor "IUESIPS" software, is not known to produce wavelength errors as a function of time or other relevant variables.

Unlike other wavelength comparisons of the $I U E$ calibration, our study relies on crosscorrelations of like spectra, that is, of the same star. Cross-correlation studies have a high internal accuracy and offer safeguards against systematic errors in the measurement process. However, they also have the disadvantage of referring the measurements to a reference spectrum template for which the zero-point itself must be determined. Thus, a second goal will be to evaluate the "absolute" wavelength zero-point errors of the IUE cameras in

\footnotetext{
${ }^{1}$ NEW Spectral Imaging Processing System (see Garhart et al. 1997).
} 
high-dispersion. This assessment has been carried out with respect to instruments on other space-borne platforms, specifically the Goddard High Resolution Spectrograph; (GHRS; operational period 1990-1997) and the Space Telescope Imaging Spectrograph (STIS; 1997-present) on board the Hubble Space Telescope. We will also compare zero-points of the $I U E / S W P$ wavelength "system" with ground-based measurements of reference stars and absolute (laboratory) values compiled in the literature (Brandt et al. 1998).

A list of actual camera sequence numbers for observations used in this work are available by request from the MAST.U The spectra utilized were the "absolute calibrated" flux extensions of the "MXHI" FITS files.

\section{Procedures and Calibrations}

\subsection{Cross-Correlation Tools}

A cross-correlation analysis offers both a convenient and efficient method of determining wavelength shifts for a series of test spectra with respect to a reference spectrum. This is largely because it utilizes the aggregate signal contained in the spectrum. No pre-conditions are required in terms of the shapes or positions of individual spectral features. Thus, the presence of practical problems such as line blending or continuum placement do not degrade the accuracy of the solution as long as these as errors are independent of wavelength. Even a limited signal-to-noise ratio will decrease the precision of the result, but it does not introduce a bias. To implement the cross-correlation analysis we used the IUEDAC

\footnotetext{
${ }^{2}$ Multi-Mission Archive at the Space Telescope Science Institute, funded through a NASA contract to the STScI.
} 
p IDL routine CRSCOR. This program is itself a "wrapper" for a small IDL collection of programs. It computes the cross (auto) correlation function according to its definition: that is, by shifting iteratively a test spectrum with respect to the reference by a series of regularly spaced wavelength increments and computing the sum of the product of the flux differences of the shifted and unshifted spectrum at each pixel within a specified wavelength or velocity range. This maximum of this function is located by least-squares polynomial fitting. For echelle data the natural unit is velocity, and so the pixel shift produced by the program is converted to $\mathrm{km} \mathrm{s}^{-1}$ as an output option. As implemented, CRSCOR truncates the start and ending wavelengths of the spectral array to the same values, thereby avoiding the generation of any false "noise" due to wrap-around of shifted arrays. The program interpolates the test spectrum to the wavelength grid of the reference template to accommodate subpixel wavelength shifts. This step also facilitates comparisons with spectra from other instruments. To test these interpolations we cross-correlated several test spectra against copies of themselves by using a similar (but not identical) set of wavelengths from other observations of the same target. This comparison tests the accuracy of interpolating spectra observed at discrete wavelengths. We found that interpolations to one set of wavelengths produce false shifts of always less than $\pm 1.0 \mathrm{~km} \mathrm{~s}^{-1}$.

Our procedure was to run $C R S C O R$ iteratively for each echelle order and to evaluate the net shift of the order as a whole wavelength segment. In our procedures we took care to screen the data for known pathologies of the IUE cameras. For example, fluxes near the edges of the order (particularly the long-wavelength end for the SWP camera) were avoided because the noise is high and also because systematic errors can be large in the blaze "ripple correction" (Gonźalez-Riestra et al. 1998). Such errors often mean sloped continua, which

\footnotetext{
${ }^{3}$ IUE Data Analysis Center (IUEDAC) was a group in the IUE Project dedicated to writing specialized IDL software for the analysis of IUE spectra.
} 
can lead to a small bias in line centroid positions. We also excluded small groups of pixels with negative data quality flags (usually associated with instrumental "reseaux") because their fluxes are generally meaningless. In these cases, we interpolated the fluxes from neighboring pixels. This step has the effect of adding incrementally to random noise but does not introduce a random shift unless the interpolated regions in the two spectra being compared are extensive and have different slopes. Apart from specific tests on zero-point shifts of interstellar line systems (\$4.) discussed below, we did not exclude interstellar lines from our cross-correlations. Although these lines are formed in a velocity frame shifted from the photospheric lines, the resulting wavelength shift can be assumed to be the same for different instruments because the cross-correlation routine does not distinguish between features formed in various velocity frames. For example, we did not have to exclude "wind" lines because the shifts from orders including these lines did not differ noticeably from shifts of adjacent orders.

In comparing IUE spectra with spectra from the HST GHRS and STIS instruments, we convolved the HST data by gaussian broadenings to make their resolutions equivalent to $I U E$ spectra. When all these steps were carried out, we experimented with $C R S C O R$ in an interactive mode for the investigations discussed in $\$ 4$. After finding that shifts arising from unflagged data pathologies are rare, we automated the program in order to compare large groups of spectra efficiently.

\subsection{The Calibration of Wavelengths in NEWSIPS}

Before an analysis of the zero-point errors of wavelengths in the IUE cameras can proceed, it is necessary to review the steps taken in the wavelength calibration of NEWSIPS data; some of the following details have not been published in any form. The 
calibration of wavelengths in an echelle system requires the prior mapping of pixels from a two-dimensional detector surface to a series of one-dimensional arrays each of which is represented as a smoothly varying function of wavelength. In the raw detector geometry the echelle orders fall at unique angles relative to the electronic scan axis, and thus together they run nonparallel to one other. In addition, in certain places on the detector the spectral format can be displaced by local shears from slight nonuniformities within the optical coupling elements. All of these factors necessitate a detailed geometrical resampling of the raw echellogram image to a new surface in which the orders run parallel along a common dispersion axis and in which their fluxes have been reevaluated at a constant velocity increment. NEWSIPS maps the raw positions to a rectilinear geometry, thereby simplying the extraction of spectral fluxes (for details see Chapters 7-9 of Garhart et al. 1997). Wavelength calibration ("WAVECAL") observations of the three cameras were made monthly during the mission through the small aperture by means of on-board platinum-neon (Pt-Ne) lamp source. Early in the mission a tungsten flood lamp ("TFLOOD") exposure was added to the emission-line exposure in order to enable process software to determine the positions of reseau more easily. In April, 1990 this practice ceased when studies within the IUE Project demonstrated that these pre-exposures added a noise component to the signal which effectively reduced the instrumental dynamic range and thus the number of measurable emission lines.

With the linearized fluxes extracted, the wavelength system of each spectrograph camera could be calibrated by means of a reference calibration spectrum by remapping the $(x, y)$ positions of its emission lines from the original geometry to the rectilinear detector surface. For this step one may think of the dispersion axis as " $x$ " and of the spatial (or echelle order $m$ ) axis as " $y$." Wavelengths of the emission lines were taken from the study of Reader et al. (1990), which was commissioned for the purpose of calibrating wavelengths of the HST spectrographs. The first of four steps in the NEWSIPS wavelength calibration 
procedure was interactive and consisted of running the IRAF母 routine ecidentify to map the positions of a set of lines to wavelengths for a reference $W A V E C A L$ echellogram.尸 In this step a quadratic Cheybshev polynomial functon was used to map the $(x, y)$ pixel locations in the reference echellogram to a wavelength table. The second step was to utilize the IRAF routine ecreidentify Chebyshev solution determined for the reference echellogram in to determine predicted pixel positions for a few hundred lines in all the WAVECAL observations for a given camera. A polynomial fit was performed for each of the echellograms, resulting in a set of wavelength zero-points and mean pixel-to-pixel wavelength increments for each order. In practice, the wavelength zero-points for each "science echellogram" were fit to a cubic polynomial in both time and camera-head temperature ("THDA"). These fits were adopted for all science data but of course not for the WAVECAL calibration observations themselves. This last step compensated for image shifts statistically by means of a previously derived least squares correlation of wavelength shift as a function of time and temperature. Note that the wavelength calibration of the WAVECALs technically applies to observations made through the small aperture. However, this calibration can be readily applied to large-aperture observations from prior measurements of the offset between the two apertures in the dispersion direction.

We should pause to point out that whereas the wavelength calibration for NEWSIPS was performed with a simultaneous polynomial fit in both the wavelength and echelle order dimensions, the number of WAVECAL emission lines was sufficient to permit a calibration

\footnotetext{
4 "IRAF" is distributed by the National Optical Astronomy Observatories, which are operated by the Association of Universities for Research in Astronomy, Inc., under cooperative agreement with the National Science Foundation.

${ }^{5}$ The reference echellograms used in this step were SWP 38001, LWP 15422, and LWR 18381.
} 
of wavelengths for most echelle orders separately (Smith 1991). The first ("global") approach is clearly the preferred one if pixel-to-pixel spacings on the detector are regular because a large number of lines can contribute to the solution for any individual order. However, during the $I U E$ lifetime the cameras suffered both electro-optical distortions and differential shifts arising from shears between fiber-optical bundles within the coupling-plate of the camera. A global wavelength solution tends to smooth over these small-scale distortions, causing correlated order-to-order errors in derived wavelengths. Consequently, with exceptions noted by Garhart et al. (1997), the ecidentify steps described above were implemented for individual spectral orders in the NEWSIPS calibration.

\subsection{Cross-Correlation of Wavelength Calibration Spectra}

In this section we discuss various time-dependent characteristics of the WAVECAL echellograms used to calibrate the science data. It should be emphasized that most of the following results were initially derived for use in removing low-order time-trends in the science data within the pipeline NEWSIPS processing of science spectra. The characteristics for $W A V E C A L$ spectra discussed below have only an indirect effect on the systematics and accuracy of the zero-points of science observations.

Starting with the cross-correlation software described in $\$ 2.1$. we compared wavelengths for some 50 echelle orders in all small-aperture, optimally exposed WAVECAL spectra (278 for LWP, 291 for LWR, and 468 for SWP) against the reference spectra used by Garhart et al. (1997) in the ecreidentify step of their calibration. The cross-correlations showed that the zero-point shifts found for the $S W P$ data have the most complicated dependences of all three cameras and, in particular, that they exhibit fluctuations in wavelength as well as time. An example of the dissimilar dependences with time at different wavelengths for this 
camera is depicted in Figure 1 1 and requires some discussion. In analyzing the WAVECAL data, we found that it was necessary to discard the pre-1979.0 (satellite commissioning period) SWP camera WAVECALs from further analysis because the zero-points otherwise showed a very steep increase during 1978. We then fit the shifts for the remaining sample of 415 spectra with 7th-degree polynomial both in time and echelle order. No such complications were found for the long-wavelength cameras. The cross-correlation results for all three cameras are plotted with time in Figures 2 and 3. Figure 2 was constructed by averaging the wavelength shifts such as those shown in Fig. [ over echelle orders.

The complicated dependence of the $S W P$ camera wavelength zero-points as a function of time and spatial direction (echelle order) is reminiscent of the complicated decay of this camera's "null flux" surface with time, as recently documented by González-Riestra (1998) and Smith (1999). During 1979-80 the zero-point of the WAVECAL spectra increased smoothly by $\approx+15 \mathrm{~km} \mathrm{~s}^{-1}$ (about 2 pixels) for order $m=70(\lambda 1170)$, decreased by about $-4 \mathrm{~km} \mathrm{~s}^{-1}$ during the mid-1980's, and decreased rapidly during the last 2-3 years of the mission. For $m=118(\lambda 1970)$, at the other end of the camera, the corresponding changes in zero-point are $+4.5 \mathrm{~km} \mathrm{~s}^{-1}, 0 \mathrm{~km} \mathrm{~s}^{-1}$, and $-2 \mathrm{~km} \mathrm{~s}^{-1}$. One sees that the greatest changes occur in the "short-wavelength" corner of the echelle surface, primarily during both the early and late stages of the IUE mission. Since changes occurred in the raw background flux at about the same epochs and in the same region of the camera as the changes we report here, it is possible that changes in the camera characteristics influenced the positions of the echelle format as well as the net fluxes of these orders as a function of time.

In contrast, the WAVECAL zero-points for the long-wavelength cameras can be fit with low order polynomials, and they do not have a marked dependence on position on the detector. The $L W P$ camera is particularly well behaved in this respect since its wavelengths (Fig. 2a) change linearly by $+3 \mathrm{~km} \mathrm{~s}^{-1}$ over the interval 1980-1996.8. For the $L W R$ camera 
(Fig. 2b) the wavelengths change by about $+4 \mathrm{~km} \mathrm{~s}^{-1}$ during $1978-1980$ and remain almost constant thereafter.

Low-order (cubic) fits for the zero-point dependences of the WAVECALs for the three cameras were hard-coded into NEWSIPS in order to remove such trends from the science data. For the $L W P$ camera data the dependence on time is linear, so the trends could be removed completely. For the $L W R$ camera the removal of trends is probably very good except for fitting the rapid increase during the first 2-3 years of the mission. A cubic polynomial cannot fully remove this early-epoch trend. As implied above, the complex dependence for the $S W P$ camera cannot be accurately fit with a cubic polynomial function, particularly either early or late in the mission. This can be seen by comparing the cubic fit to the shifts in Figure 2. The differences between this line and the zero-points of the individual WAVECAL spectra imply that, independent of other error sources, $S W P$ data can be expected to have small $\left(\leq \pm 2 \mathrm{~km} \mathrm{~s}^{-1}\right)$ epoch-dependent errors.

Aside from temporal trends in the mean shifts, one can see from Figures 2 and 3 that the r.m.s. scatter characteristics of zero-points among WAVECAL spectra also change with time. Considering the $S W P$ camera results first, Fig. 2 shows that starting sometime in 1988-90 the scatter decreased to only about $60 \%$ of its initial value of $\pm 2.5 \mathrm{~km} \mathrm{~s}^{-1}$. Also, the statistical outlying points (defined as those differing from the epochal mean by at least 1 pixel or $8 \mathrm{~km}^{-1}$ ) decreased in occurrence from over $10 \%$ of all obervations to only about $4 \%$. It is possible that these changes are caused by the termination of adding TFLOOD lamp exposures onto the WAVECAL spectra. Other changes were made during this period, such as taking these observations under more tightly defined constraints in THDA and focus, might have contributed to the improved scatter characteristics as well. The scatter for $L W R$ WAVECAL data seems to decrease well before 1990, so the practice of adding TFLOOD does not seem to be important. Also, notice that the scatter for the $L W P$ camera actually 
seems to have increased markedly in 1990. The suddenness of this change, particularly in the LWP camera, suggests that the change in adding TFLOOD flux might have contributed to the scatter of the WAVECAL zero-points for the calibration of this camera.

\section{Systematics in IUE Parameter Space}

\subsection{Comparison of IUESIPS and NEWSIPS}

\subsubsection{Short-Wavelength Prime Camera:}

There is no doubt that for radial velocity or wavelength studies involving IUE data, the NEWSIPS processing is much superior to that of IUESIPS. The wavelength calibration coefficients were periodically updated during the mission in IUESIPS, so datasets spanning several years cannot have consistent wavelengths. In this section we quantify the zero-point differences between IUESIPS and NEWSIPS calibrations during the mission.

For this purpose we chose hot stars that the IUE observed over nearly the entire satellite lifetime. The first two stars, $10 \mathrm{Lac}(09 \mathrm{~V})$ and $\tau$ Sco $(\mathrm{B} 0.2 \mathrm{~V})$, are sharp-lined spectral standards with no known radial velocity variations. We obtained all $S W P$ high-dispersion observations from the MAST database after 1979.0 (except for SWP 01205, 01765, \& 02051 for which IUESIPS data were not available) and cross-correlated pairs of IUESIPS and NEWSIPS fluxes in spectrum segments in orders $m=50-119$. These results are shown in Figure 4 . The series of dotted vertical lines mark the dates in which then

newly-derived wavelength calibrations were implemented into IUESIPS processing. We also cross-correlated 33 echellograms from both processing systems for six white dwarfs which were analyzed by Holberg, Barstow, \& Sion (1998; hereafter "HBS"). These stars are WD 
0005+511, WD0044-121, WD0621-376, EG-102, GD-394, and WD2211-495, and in all cases

their spectral lines are sparse and broad. Thus, we found it advantageous to cross-correlate the same 50 orders of these spectra as those utilized for our study of 10 Lac and $\tau$ Sco. We also cross-correlated a few orders dominated by interstellar lines. We found a mean zero-point difference, IUESIPS - NEWSIPS, among the sample of HSB white dwarfs of $+9.5 \pm 1 \mathrm{~km} \mathrm{~s}^{-1}$. This compares rather well with the difference of $+9.1 \mathrm{~km} \mathrm{~s}^{-1}$ given by HSB (referred to $\lambda 1400)$.

The dependences of zero-point with time for both white dwarfs and hot main sequence stars in Fig. 1 are likewise similar to the HBS results. Thus, the form of this zeropointdifference curve is insensitive to the sample of stars chosen. Rather, it is largely determined by the dates of the revisions of the IUESIPS calibration constants were implemented (see vertical dotted lines in the figure). Notice that the net difference, IUESIPS - NEWSIPS, is positive for all epochs and increases during the late-1980's to a rather well determined maximum of $+18 \pm 1 \mathrm{~km} \mathrm{~s}^{-1}$ at 1992.5. It then decreases quickly during 1993 and is perhaps constant thereafter. The IUESIPS wavelength calibration was unsettled prior to 1983 , producing a large scatter compared to the homogeneously-calibrated NEWSIPS system.

\subsubsection{Long-Wavelength Cameras:}

We also compared IUESIPS-NEWSIPS wavelength zero-point differences for the two long-wavelength cameras; we believe this is the first such study of its kind. This endeavor turned out to be far more difficult because of the paucity of data over an extended time for any given star. As a result, we were unable to extract information on the time evolution of the wavelength zero-point differences for these two cameras.

To determine a IUESIPS - NEWSIPS mean difference for the two long-wavelength 
cameras, we considered the K2 III star $\alpha$ Boo as well as three of the four photometric calibration ("PHCAL") standards for the IUE mission, $\zeta$ Cas, $\tau$ Sco, and $\eta$ UMa. A number of echelle orders of spectra of the hot stars do not contain many lines in this spectral region, so we selected several orders interactively that gave a reasonable signal in the cross-correlation function and used them alone. The averages of the IUESIPS - NEWSIPS differences can be summarized as follows. For the LWP camera invesigation we obtained $+0.5 \mathrm{~km} \mathrm{~s}^{-1}$ for $\zeta$ Cas (126 spectra), $+3.2 \mathrm{~km} \mathrm{~s}^{-1}$ for $\tau$ Sco (91 spectra), $+3.6 \mathrm{~km} \mathrm{~s}^{-1}$ for $\eta$ UMa (165 spectra), and $4.6 \mathrm{~km} \mathrm{~s}^{-1}$ for $\alpha$ Boo (19 spectra). For the $L W R$ camera the corresponding differences are: $+5.9 \mathrm{~km} \mathrm{~s}^{-1}$ for $\zeta$ Cas $(65$ spectra $),+6.9 \mathrm{~km} \mathrm{~s}^{-1}$ for $\tau$ Sco (38 spectra), $+6.5 \mathrm{~km} \mathrm{~s}^{-1}$ for $\eta$ UMa (107 spectra), and $6.3 \mathrm{~km} \mathrm{~s}^{-1}$ for $\alpha$ Boo (11 spectra). Taking the straight mean of these results, we have the following differences:

$$
\begin{gathered}
\Delta \mathrm{RV}_{L W P}=\mathrm{RV}_{\text {IUESIPS }}-\mathrm{RV}_{\text {NEWSIPS }}=+3.0 \pm 2 \mathrm{~km} \mathrm{~s}^{-1} \\
\Delta \mathrm{RV}_{\text {LWR }}=\mathrm{RV}_{\text {IUESIPS }}-\mathrm{RV}_{\text {NEWSIPS }}=+6.4 \pm 0.5 \mathrm{~km} \mathrm{~s}^{-1}
\end{gathered}
$$

In these relations we have applied the (larger) r.m.s. values from the $L W P$ camera to the $L W R$ camera. The shift for the $L W R$ camera is almost certainly significantly different from zero. As with the $S W P$ camera, the difference(s) are likely to arise from periodic changes in the IUESIPS wavelength calibration.

\subsection{Zero-Points Errors between Large and Small Apertures}

Each of the three IUE cameras included a pair of science apertures in the telescope plane, a small ("SMAP") and large (" $L G A P$ ") aperture. The SMAP was used primarily during the first few years of the mission because of concerns about the wavelength stability for large-aperture observations. Thus, acquisition/guiding errors might result in light losses, but they would not so seriously affect wavelength accuracy as they would for the LGAP. 
With experience in acquiring objects in the Fine Error Sensor (FES), the IUE Project realized that better wavelength precisions could be obtained than initially anticipated, and observations through the large aperture eventually became routine. A related operational issue was the different centroiding modes in which the FES was used to acquire targets prior to guiding. In particular, the so-called "underlap" and "overlap" modes were to acquire bright and faint stars, respectively. Although the underlap mode in principle causes an offset in the target position, pains were taken to correct for this offset. To optimize these complimentary acquisition strategies, the Project settled on a suitable magnitude demarcation of $\mathrm{m}_{v}=4.5$. Thus, the different acquisition strategies can lead potentially to differences in the wavelength scale between bright and faint stars.

To evaluate aperture-to-aperture differences, we proceeded to evaluate shifts from cross-correlations of similar spectra. We chose NEWSIPS-processed spectra from several well observed $\mathrm{OB}$ and white dwarf stars. Table — gives the numbers of IUE high-dispersion echellograms used for the analysis of the $S W P$ camera data. As before, these data were cross-correlated for echelle orders $m=70-119$. The zero-point errors are expressed in velocity units, a represention that we justify in $\$$ 4.1. Table 1 shows that both the mean differences, expressed as $\mathrm{RV}_{L G A P}-\mathrm{RV}_{S M A P}$ velocities, are $-0.8 \mathrm{~km} \mathrm{~s}^{-1}$ for six bright stars and $-0.7 \mathrm{~km} \mathrm{~s}^{-1}$ for six white dwarfs. The mean shift for any one of these stars is accurate to about $\pm 2 \mathrm{~km} \mathrm{~s}^{-1}$ or better. Thus, there are no noticeable systematic differences for shifts between the two apertures and between the over-/underlap modes guiding modes.

Table 1 lists similar results for three stars for the $L W P$ camera and four stars for the $L W R$. The mean $L G A P$ - SMAP difference for the two cameras are $-1.2 \pm 3$ and $+0.2 \pm 3$ $\mathrm{km} \mathrm{s}^{-1}$, respectively. The quoted errors are sums (added in quadrature) of both LGAP $S M A P$ differences and zero-points of individual $S M A P$ observations. From this work it is clear that no major systematic aperture-to-aperture differences are detectable for any of 
the three cameras.

\subsection{Dependence of Zero-Point with Time}

One may use the large $S W P$ camera datasets just discussed to determine if trends are present in science observations with respect to time. Table 1 exhibits the linear regression slopes with time for the large-aperture data for the three cameras. The data represented are in most cases the same as in Table 17. The second row of each data group lists the ratio of the regression slope to the r.m.s. error $\sigma$ in the slope. For the $S W P$ data the regressions for three stars are significant to at least three sigma. For one of these three stars, $\zeta$ Oph, the non-zero slope was caused mainly from data by an intensive campaign conducted by one observer. Investigation showed that these differences could be traced to use of a different reference position in the FES. Thus, the derived slope for this star is questionable.

Unlike the case of $\zeta$ Oph, the $S W P$ data for two well-observed stars, HD 60753 and $\mathrm{BD}+75^{\circ} 325$ in Table 2, show clear negative trends with time (see Figure 5) that cannot be readily attributed to observational practices or instrumental conditions. A similar trend may also be present in the data of the A5 V star HD 11636 (not shown), for which the far-UV fluxes are faint. We might speculate that this apparent trend is actually the norm for UV-faint sources. For example, it is possible that these trends, if real, are by-products of an unexplained source of scattered light, i.e., "the streak," which appeared in 1991. In fact, the scattered light from this artifact introduced a gradient in the light admitted across both the small and large apertures required that IUE telescope operators to adopt a new acquisition reference point in the FES to avoid shifts in the spectra of several $\mathrm{km} \mathrm{s}^{-1}$ (Pitts 2000). This displacement is similar to the change in zero-points documented for HD 60753 and $\mathrm{BD}+75^{\circ} 325$. Overall, we find that all but one of the 11 stars in the sample represented 
in Table 2 shows a negative slope. When subjected to a statistical "Sign Test" (Dixon and Massey 1951)," the signs of these slopes are weakly significant at the $2 \sigma$ level. We conclude that over a 15 year interval a trend of several $\mathrm{km} \mathrm{s}^{-1}$ might exist for faint objects. We stress that this trend is not apparent in our bright objects results.

In contrast to the $S W P$ camera results, we have found no clear velocity trends for spectra observed with the $L W P$ and $L W R$ camera. The $L W P$ sample consisted of observations of eight stars: $\tau$ Sco, 10 Lac, $\eta$ UMa, $\zeta$ Cas, HD 93521, RR Tel.冋 BD+28²11, and $\mathrm{BD}+75^{\circ} 325$. The distributions of shifts of all these datasets showed equal numbers of positive and negative slopes with time, none of which were significant above the level of $1.5 \sigma$. The occurrence in October 1983 of a large "flare," a developing hole in the high voltage assembly, thereby producing a high, localized background, complicated the analysis of late-epoch data for this camera. The flare caused the IUE Project to reduce the camera voltage, resulting in a loss of sensitivity. This sequence of events led to a curtailment in the camera's use and an abbreviated time interval used to monitor instrumental trends of well observed objects. Among our sample of program bright stars and white dwarfs, we were able to find only two stars, $\zeta$ Cas and $\tau$ Sco, with an appreciable number of same-aperture observations before and after the flare. Of these two targets, $\zeta$ Cas is the better sampled in time. Its cross-correlation shifts exhibited no trend with time, but they did exhibit a

\footnotetext{
${ }^{6}$ The UV spectrum of RR Tel, a symbiotic binary, is dominated by several strong emission lines. The cross-correlation results we report are for those orders containing these emission lines. Since these orders contain only 1-2 strong lines apiece the r.m.s. errors are large, \pm 6.5 $\mathrm{km} \mathrm{s}^{-1}$. Other orders in these spectra contain faint absorption lines, which produce an r.m.s. in their shifts of nearly the same value, $\pm 7.1 \mathrm{~km} \mathrm{~s}^{-1}$. The orders containing emission lines give comparable quality cross-correlation results as those without them. RR Tel shows by far the poorest cross-correlations of any spectra in our sample.
} 
pronounced increase in scatter (about a factor of two) after 1984.0. The data for $\tau$ Sco

showed an apparent slope of $-1 \mathrm{~km} \mathrm{~s}^{-1} \mathrm{yr}^{-1}$, but this could be an artifact of a greatly increased scatter after 1984.0. An examination of the shifts as a function of wavelength for this star revealed no wavelength-dependence. This would be surprising if the flare were the cause of wavelength shifts. Our conclusion from this investigation is that the wavelength zero-points of $L W R$ observations taken after the 1983 flare event are less reliable than before. However, this may not be a direct consequence of the flare itself. Rather, we suspect that the increased scatter arises from the decreased usage of the camera and the resulting lower accuracy of the necessary redetermination of positions in the FES used to acquire an object.

\subsection{Dependence with Focus and Temperature}

Early in the IUE mission it was discovered that the camera image format shifts frequently in both the spectral and spatial directions with time and camera temperature. Camera temperature was measured roughly by thermistors located in any of several areas of the camera head amplifier (not an ideal location to measure the temperature of the spectrograph bench itself), and one of these was selected to provide a consistent refererence. This provided a "THDA" index which could be used to calibrate wavelength zero-point shifts and apply them to the processing of a particular observation in IUESIPS and NEWSIPS. Motion of the spectral format on the detector was generally caused by telescope flexures and, under routine operating conditions, secondarily by changes in electro-optical properties of the camera. Thus, fluctuations in satellite temperature (which was primarily a function of the telescope's orientation angle with respect to the Sun) caused the telescope to expand or contract and the focus to change. The focus was usually controlled indirectly by turning on or off a heater located behind the primary mirror. An alternate control 
procedure consisted of toggling a heater mounted on the spectrograph deck. Although the camera temperature and detector image shifts were correlated, a hysteresis arising from the instrument's thermal history tended to weaken the efficacy of controlling the focus by commands to the spacecraft. This control was weakened further by other environmental factors, such as the Earth's eccentric orbit around the Sun and earthglow. Consequently, the IUE Operations team developed a conservative strategy of maintaining the THDA temperature within certain limits. During the course of the IUE mission statistical correlations were derived from shifts of WAVECAL spectra as a function of the THDA and focus parameters. These shifts were parameterized by means of a polynomial fit, so NEWSIPS could remove them to first order in its assignment of wavelengths.

As part of our cross-correlation analysis, we searched for a correlation between zero-point shifts and THDA and focus values at the time of an observation. Generally, we were unable to find a convincing correlation. Except for an accident of an unrelated contemporaneous research program, this search might have stopped with this null result. However, while investigating spectral variations in B stars for another research program, we used the cross-correlation tools from the present study to shift spectra to the same wavelengths before manipulating the data further. In doing so, we noticed a time-dependent cycle of about a day (see Figure 6) in the results for a time series of 22 observations conducted in 1995 on the B3Ve star $\alpha$ Eri. In searching through the IUE archival database further, we found an extraordinary set of 181 continuous observations on the B0.5V star $\epsilon$ Per in 1996 obtained by Dr. D. Gies. Figure 7 shows that the same one-day cycle is present in the data for this star. A string of observations of HD 93521 at 1994.2 (not shown) exhibits a similar 1-day period and few $\mathrm{km} \mathrm{s}^{-1}$ semiamplitude over three days.

Because the cycle lengths in these shifts are all close to one day, we investigated first whether the satellite's orbital motion might have somehow been neglected in determining 
wavelengths for an individual observation. However, we were able to discount that possibility.

A more successful attempt to explain the apparent 1-day velocity cycles of $\alpha$ Eri, $\epsilon$ Per, and HD 93521 was to investigate the effects of varying the instrument temperature by adjusting the telescope focus. Cycling the camera-deck heater produced correlated responses of the THDA and telescope-focus values, especially when the ambient spacecraft temperature was lower than nominal, as for the 1996 observations of $\epsilon$ Per. During this particular monitoring series, the good correlation between temperature and focus indicates that the focus was controlled by the deck heater. In the time series on $\alpha$ Eri the temperature was not quite so low. Then an adequate means of controlling the focus was to cycle the more distant telescope mirror heater. Since the telescope heater was isolated from the spectrograph, the locally measured THDA value did not correlate well with the focus changes of the telescope (see lower dashed line in figure). However, in either case the principle was the same, that heating could be applied to prevent the focus from drifting to large negative values. The important point is that this application caused an overcorrection of the focus. The focus values would then swing (relatively) positive until a low ambient temperature again reversed the change of the focus, causing a new thermal-focus cycle to ensue. All told, the changes in focus from either thermal-control technique caused the velocity to become first too negative and then too positive by a few $\mathrm{km} \mathrm{s}^{-1}$. Although we have found the velocity-focus correlation only in $S W P$ camera datasets so far, it is probably present in long-wavelength data as well.

Figure 8 exhibits the correlation explicitly between telescope focus value and zero-point for the observing campaign on $\epsilon$ Per. A similar plot can be constructed for THDA instead of focus, but the $\alpha$ Eri data imply that the correlation of velocity with THDA for the $\epsilon$ Per data is a result of temperature excursions changing the focus, and not a shift caused by 
the temperature variation within the camera. Note especially that Fig. 8 shows that the correlation arises only in the limited focus range of -2.0 to -3.7 (instrumental units). As noted above, we also searched our results for a dependence on focus and THDA values in our study of time-dependent correlations, but we found none. We suspect the reason is that IUE observations of most objects were obtained at different epochs and with different target-centering practices. This would tend to conceal any correlation over a small critical subrange of focus values in our searches for trends in the stars of Table 11. However, we expect the pattern shown in Fig. 8 is present in all high-dispersion data to some extent. If so, it is a a secondary source of radial velocity error.

\section{Radial Velocity Zero-Point Errors}

\subsection{Wavelength-Systematics?}

In our earlier discussion we focused only the temporal results because we did not find any systematic dependences on wavelength. In Figure 9 we demonstrate this further by exhibiting the averages and confidence belts $( \pm 1 \sigma)$ of shifts for 16 stars at each wavelength interval in our $S W P$ camera study. The mean r.m.s. over wavelength for all stars is \pm 2.4 $\mathrm{km} \mathrm{s}^{-1}$ while the adjacent order-to-order shift is only $\pm 1.0 \mathrm{~km} \mathrm{~s}^{-1}$. These errors are generally wavelength-independent except below $\approx \lambda 1230$. Below this wavelength the errors

increase because the detector loses its sensitivity to flux. Because there is no clear trend in wavelength, we will refer to the zero-point error in terms of apparent velocity rather than wavelength.

A comparison of the velocity fluctuations resulting from the cross-correlations among different stars suggests that the precision of a zero-point determination varies as a weak 
power of the reciprocal of the net flux (detector counts). In addition, we find that order-to-order shifts are measurably larger for spectra of white dwarfs and rapid rotators (e.g., $\eta \mathrm{UMa}, \zeta$ Cas). This fact suggests that the random cross-correlation errors increase

slightly with line broadening, namely from about $\pm 3 \mathrm{~km} \mathrm{~s}^{-1}$ (e.g., $\tau$ Sco) to $\pm 4 \mathrm{~km} \mathrm{~s}^{-1}$ (e.g., $\eta \mathrm{UMa})$.

\subsection{SWP Analysis}

\subsubsection{IUE versus GHRS}

a) Available Short-Wavelength Atlases

Since the completion of the processing of $I U E$ data with NEWSIPS in 1997, new spectral atlases have been constructed using the $G H R S$ wavelength calibration. Both the IUE/NEWSIPS and GHRS wavelength calibrations are derived from a recent homogeneous study of ultraviolet platinum lines (Reader et al. 1990). The most important atlas for purposes of comparison to $I U E / S W P$ data is the Brandt et al. (1998) atlas of 10 Lacertae. This work covers the region $\lambda \lambda 1181-1777$, which is most of the wavelength range covered by the SWP camera $(\lambda \lambda 1160-1980)$. In order to insure that image wandering could not contribute significantly to errors, all observations for this atlas were made through the GHRS Small Science Aperture. Aside from being a spectral standard (Walborn 1972), $10 \mathrm{Lac}$ is an ideal object for calibrating the wavelength scale. 10 Lac is an apparently single star, and its spectrum has numerous sharp photospheric lines. Fullerton (1990) found no measurable variations over time to a precision of perhaps $\pm 1 \mathrm{~km} \mathrm{~s}^{-1}$. In addition to plotted specta, the Brandt et al. atlas gives measured wavelengths of over 700 lines, 
which can be compared to published laboratory values.] This information is adequate to permit the determination of an effective mean velocity of the IUE SWP camera relative to ground-based velocity measurements of 10 Lac (Hobbs 1969, Grayzeck \& Kerr 1974, Stokes 1978).

b) The Procedure

In this subsection we will link the apparent velocities of SWP LGAP spectra of 10 Lac to the laboratory system. Because this connection takes several steps, we will first outline them and then discuss the actual cross-correlation results:

Step 1, $\Delta R V_{S W P}=R V_{S W P-L G A P}-R V_{S W P-S M A P}$ :

This is a velocity correction for unpredicted difference in wavelengths of lines measured in IUE spectra taken through the large aperture relative to the small aperture. Such errors might arise from potential measurement error between the apertures or (more likely) from changes in the FES reference position used for target acquisition.

Step 2, $\Delta R V_{I U E}=\Delta R V_{S W P-L G A P}-R V_{G H R S}$ :

This term is the instrument-to-instrument correction for the SWP/SMAP wavelength zero-point referred to the zero-point of the GHRS (small-aperture) atlas of 10 Lac.

Step 3, $\Delta R V_{U V}=\Delta R V_{G H R S}-R V_{l a b}$ :

This correction accounts for any difference between the radial velocity derived from differences in the measured $(G H R S)$ and laboratory wavelengths. These could result from an error in the calibration of the GHRS. We note in particular that the Brandt et al. wavelengths of photospheric lines in 10 Lac were measured from mainly iron-group element

\footnotetext{
${ }^{7}$ The full table of measured and laboratory wavelengths used by Brandt et al. is available at the URL http://archive.stsci.edu/hst/atlas10lac/datalist.html.
} 
lines whereas the calibration of wavelengths for the GHRS systems was derived from Pt line wavelengths. [

Step 4, $\Delta R V_{\text {star }}=R V_{\text {lab }}-\Delta \mathrm{RV}_{\text {optical }}$ :

The final step is the correction for the star's adopted heliocentric radial velocity from ground-based measurements. The ground-based value can be compared finally to the velocity obtained from appying Steps 1-3. In our chain of comparisons, the defining reference is the radial velocity of $10 \mathrm{Lac}\left(-9.7 \mathrm{~km} \mathrm{~s}^{-1}\right.$; Wilson 1953). The addition of the results from Steps \#1-4, $1+2+3+4$, gives the net discrepancy between the SWP LGAP and the laboratory wavelengths.

c) Comparison with 10 Lacertae Atlas

Analysis for Step 1, $\triangle R V_{S W P}$ (correction from IUE large to small aperture):

In $\$ 3$. we showed that $S W P$ camera data for several bright hot stars do not have discernible velocity trends with time. As Figure 10 shows, the test star, 10 Lac, is no exception. The mean aperture-to-aperture difference from all available 10 Lac spectra is $\Delta \mathrm{RV}_{S W P}=+0.4 \mathrm{~km} \mathrm{~s}^{-1}$. At $+1.2 \mathrm{~km} \mathrm{~s}^{-1} \pm 0.8 \mathrm{~km} \mathrm{~s}^{-1}$, this value differs slightly from the average velocity discrepancy for 12 stars represented in Table 11.

As an important digression, we should add that we have searched for nonrandom positionings of the image of 10 Lac within the LGAP. Figure 11 exhibits the histogram of the SWP/LGAP sample of spectra. The centroid of this distribution is arbitrarily set to zero. If one first fits the distribution to a single gaussian, the r.m.s. dispersion is 4

\footnotetext{
${ }^{8}$ The line list for SWP camera data was culled to 288 lines from the Reader et al. (1990) list. This is about twice the number of lines used for the IUESIPS calibration. The line list for high-dispersion LWP/LWR data contains 479 lines.
} 
$\mathrm{km} \mathrm{s}^{-1}$. A more convincing representation is a bimodal gaussian (see figure) with $\sigma_{1}, \sigma_{2}=$ $2.8 \mathrm{~km} \mathrm{~s}^{-1}$ per observation. The corresponding errors in the means of the two peaks are $0.5 \mathrm{~km} \mathrm{~s}^{-1}$ and $\pm 1.2 \mathrm{~km} \mathrm{~s}^{-1}$, respectively. Investigation of the times of the observations shows that most of the secondary peak arises from spectra taken in 1993 and 1995, when different aperture reference points were used to acquire the star. It is possible that target acquisitions at other reference points may be reason for the skewed high-velocity tail of the distribution. Departures from gaussian distributions are common among apparent velocities found from our large data samples, and the extended negative and positive tails that they represent in a histogram seem to be present equally. It may be significant that the distribution of cross-correlation shifts taken from the 1996 data series for $\epsilon$ Per, with the sinusoidal component removed, generally follows a gaussian distribution and in particular has no extended tails. This fact suggests that the effective position of the star image in the aperture is the primary reason for outlying velocities in our cross-correlation results. In this picture the initial exposures of a target in a time sequence are likely to exhibit a large scatter because the telescope temperature and focus had not yet equilibriated. After an hour or two the error distribution of the remaining observations became tighter and also more closely resembled a gaussian.

Analysis for Step 2, $\triangle R V_{I U E}$ (correction from IUE to GHRS):

Cross-correlation shifts were computed as before among 41 spectral orders in six $S W P$ SMAP echellograms against the Brandt et al. atlas of 10 Lac. These shifts are indicated by the solid line in Figure 12. The average, $\Delta \mathrm{RV}_{I U E}$, is $+0.6 \mathrm{~km} \mathrm{~s}^{-1}$, while the r.m.s. error per observation is $\pm 1.5 \mathrm{~km} \mathrm{~s}^{-1}$. This gives an error in the mean velocity determination of $\pm 0.2 \mathrm{~km} \mathrm{~s}^{-1}$. This value is negligible compared to errors from the other steps.

Analysis for Step 3, $\Delta R V_{U V}$ (correction from GHRS to laboratory system):

Brandt et al. (1998) selected 24 photospheric lines from the total sample of 705 lines 
they identified in their atlas and measured a mean velocity difference of $-7 \mathrm{~km} \mathrm{~s}^{-1}$ with respect to laboratory wavelengths in the literature. However, these authors did not specify which lines they used. We have compared the wavelengths of the entire dataset and find instead a mean velocity difference of $-11.6 \mathrm{~km} \mathrm{~s}^{-1}$. We repeated this comparison by taking a number of subsamples of lines. These differences were found to lie always within $\pm 2 \mathrm{~km} \mathrm{~s}^{-1}$ of the latter value. We adopt this value for $\Delta \mathrm{RV}_{U V}$.

Analysis for Step 4, $\Delta R V_{\text {star }}$ (correction for 10 Lac's heliocentric radial velocity):

The optical catalog radial velocity value for $10 \mathrm{Lac}$ is $-9.7 \mathrm{~km} \mathrm{~s}^{-1}$ and requires no discussion. The sign of this radial velocity will be reversed below to refer the velocity system to the $G H R S$ scale. From the agreement of values for this star from different instruments in the literature, a reasonable error for the optical determination is $\pm 1 \mathrm{~km} \mathrm{~s}^{-1}$.

In summary, the combination of the four velocities gives a net error of $=+0.4+$ $0.6-11.6+9.7=-0.9 \pm 3.5 \mathrm{~km} \mathrm{~s}^{-1}$. This is the computed discrepancy for all measured photospheric wavelengths of 10 Lac from SWP LGAP observations referenced to the UV laboratory wavelengths used in the Brandt et al. study. The error of $\pm 3.5 \mathrm{~km} \mathrm{~s}^{-1}$ is the sum added in quadrature of the values given for each of the above four steps. This mean discrepancy appears as the first entry in Table 3 and is the key single result of this paper.

\subsubsection{Alternative Atlases}

Less precise comparisons can be made between IUE SWP camera data and the panchromatic HST/STIS atlas of Arcturus (Ayres, priv. comm.) and the $H S T / G H R S$ atlas of Procyon (Wood et al. 1996). Wood et al. measured Procyon atlas lines by detailed centroid-fitting algorithms. The results were referred to an "absolute" (ground-based) scale, with a minor barycentric correction being applied for the star's orbital motion around a 
companion. These authors also noticed a systematic trend of emission line velocities with excitation potential such that the velocity of the lower-excitation features agrees with the ground-based scale within $1 \mathrm{~km} \mathrm{~s}^{-1}$. The cross-correlation with the Arcturus atlas features was carried out for two available SWP echellograms and for eight echelle orders at the long-wavelength end of this camera's range. We measured a mean IUE - STIS velocity differences of -2.2 and +0.8 (both $\pm 5 \mathrm{~km} \mathrm{~s}^{-1}$ ), respectively, giving a mean of $-0.7 \pm 5$ $\mathrm{km} \mathrm{s}^{-1}$; see Table 3. The comparison with the Procyon atlas could be made with only six observations. Again, a flux deficit at short wavelengths and a general paucity of lines restricted our cross-correlations to echelle orders 72-76. The mean IUE - GHRS difference from this comparison was $-4.9 \pm 5 \mathrm{~km} \mathrm{~s}^{-1}$. When corrected by $+1.0 \mathrm{~km} \mathrm{~s}^{-1}$ (Irwin et al. 1992), this result becomes $-3.9 \mathrm{~km} \mathrm{~s}^{-1}$ (Table 3). Errors of $\pm 5 \mathrm{~km} \mathrm{~s}^{-1}$ (taken from the comparison of IUE/SWP data to the Procyon atlas) were applied to this comparison. In this case, the data quality were higher, but there were fewer spectra in the sample. The differences from comparisons with the two atlases, $-3.9 \pm 5 \mathrm{~km} \mathrm{~s}^{-1}$ and $-0.7 \pm 5 \mathrm{~km} \mathrm{~s}^{-1}$ are consistent with the value of $-1 \mathrm{~km} \mathrm{~s}^{-1}$ determined from the 10 Lac study.

\subsubsection{Comparison of 10 Lac with stars of similar spectral type}

Another check on the results of $\S 3.1$ is to cross-correlate the IUE SWP/LGAP spectra of 10 Lac with stars of similar spectral type. Such comparisons check for target-centering errors of the reference star $10 \mathrm{Lac}$ as well as propagation of errors in the $I U E / S W P$ and GHRS calibrations (i.e., a combination of errors arising from Steps 1 and 2). Two stars which provide a ready comparison with 10 Lac are HD 93521 (O9 Vp) and HD 60753 (B2 III). We were able to cross-correlate the shifts between 10 Lac and these two stars using nearly all available spectra (at least 93 exposures in each case). To do so, we again used echelle orders $m=70-119$, except for $m=103,106$, and 109, which are dominated by 
interstellar features. Correcting for differences between the optically-determined heliocentric radial velocities for the two stars (Wilson 1953), we determined apparent radial velocity differences, in the sense "HD-star" versus $10 \mathrm{Lac}$, of $+1.8 \mathrm{~km} \mathrm{~s}^{-1} \pm 3 \mathrm{~km} \mathrm{~s}^{-1}$ and -0.5 $\mathrm{km} \mathrm{s}^{-1} \pm 3 \mathrm{~km} \mathrm{~s}^{-1}$, respectively (see Table 3). The errors listed are given by assuming that the errors inherent in each of the steps described above for 10 Lac are similar for these stars and that they add in quadrature.

\subsection{Analysis of LWP Camera Data}

Although the GHRS atlas of 10 Lac offers an excellent reference template for the IUE SWP camera, there is no spectral atlas of a nonvariable star which covers the long-wavelength camera range $(\lambda \lambda 2000-3000)$ region and has a comparable quality. Thus, we had to resort to alternative strategies to evaluate zero-point errors in long-wavelength camera data. Although it is possible in principle to cross-correlate echelle orders for $S W P$ and long-wavelength camera data over their overlapping wavelength range, practical difficulties intervene when carrying out this operation. One of these is that a loss of camera sensitivity in these regions causes a steep gradient in signal-to-noise ratio along the spectrum. In order to compare spectral lines of comparable quality, this constraint further limits the already narrow wavelength interval. In addition, spectra of even cool stars do not clearly exhibit many features. Despite these limitations, we were able to compute reliable cross-correlation shifts for the sharp-lined stars 10 Lac and $\tau$ Sco for wavelengths ( $\lambda 1949-1959)$ in common with the two cameras, namely order $m=71$ for $S W P$ and $m=118$ for $L W P / L W R$. For a comparison with the $L W R$ camera, we find apparent radial velocity differences, $\mathrm{RV}_{S W P}-\mathrm{RV}_{L W R}=-0.1$ and $-3.0 \mathrm{~km} \mathrm{~s}^{-1}$ for $10 \mathrm{Lac}$ and $\tau$ Sco, respectively. The

corresponding results with the $L W P$ camera are $\mathrm{RV}_{S W P}-\mathrm{RV}_{L W P}=+1.1$ and $+5.4 \mathrm{~km} \mathrm{~s}^{-1}$, respectively. As usual, these values refer to means of the ensembles of many $L G A P$ spectra 
for each star. We estimate the errors in these comparisons to be about $\pm 4.4 \mathrm{~km} \mathrm{~s}^{-1}$ by adding in quadrature the typical order-to-order fluctuations $\left( \pm 3 \mathrm{~km} \mathrm{~s}^{-1}\right)$ with the average velocity difference $\left( \pm 3.2 \mathrm{~km} \mathrm{~s}^{-1}\right)$ between large- and small-apertures. Since the derived zero-point differences are comparable with this r.m.s., our comparison suggests that there are no significant wavelength differences in the $\lambda \lambda 1950-2050$ region between the $S W P$ and long-wavelength camera data.

An alternative method of evaluating mean radial velocity errors for the long-wavelength errors is to cross-correlate the fluxes of $I U E$ spectra with those of $H S T$ atlases of Procyon and Arcturus. Note that the suitability of this technique relies upon our previously tying the zero-points of these atlases to the 10 Lac atlas via IUE data. This method is reliable in principle, but in practice it has the drawback that rather few IUE observations are available for these particular stars. In the case of $L W R$ camera for Arcturus, we were able to cross-correlate 9 orders in just three spectra and found a difference, $\mathrm{RV}_{L W R}$ $\mathrm{RV}_{\text {STIS }}$, of $+2.7 \pm 4 \mathrm{~km} \mathrm{~s}^{-1}$. These quoted errors and others quoted below include both the r.m.s. velocities for individual orders and the r.m.s. arising from the small numbers of observations. We cross-correlated seven available spectra with the Procyon atlas and found a difference of $-3.5 \pm 6 \mathrm{~km} \mathrm{~s}^{-1}$. A comparison between the Arcturus atlas and the $L W P$ datasets is not possible because the IUE observations were made with the star image placed in various "nonstandard" positions in the large aperture. For the Procyon atlas (3 orders; $4 L W P$ spectra) the difference, $\mathrm{RV}_{L W P}-\mathrm{RV}_{G H R S}$, is $+7.9 \pm 6 \mathrm{~km} \mathrm{~s}^{-1}$. However, this difference is noticeably affected by a wavelength shift of one spectrum (LWP 13112) with respect to the three. Although we could find no a priori reason to exclude the LWP 13112 spectrum from our analysis, its omission would result in an $\mathrm{RV}_{L W P}-\mathrm{RV}_{G H R S}$ difference of $+2.4 \mathrm{~km} \mathrm{~s}^{-1}$. This would bring the $L W P$ scale fully into agreement with the essentially null difference found in the preceding paragraph. Additionally, we compared the mean zero-point difference between the $L W P$ and $L W R$ cameras by cross-correlating many spectra 
in 59 orders of six stars. These results are summarized in Table 6 . The entries in this table give a mean difference of only $+0.4 \pm 3 \mathrm{~km} \mathrm{~s}^{-1}$, which is shown as the last entry in Table 3 . This agreement suggests again that the zero-points of these two cameras agree to within errors of about $\pm 5--6 \mathrm{~km} \mathrm{~s}^{-1}$.

To summarize all these comparisons, the radial velocity differences of the three IUE cameras, inter alia, are zero to within the measurement errors.

\subsection{Radial Velocity Zero-Point Errors: Dependence on Wavelength}

Because wavelengths were measured individually for more than 700 lines in the 10 Lac atlas, it was possible to search for wavelength errors as a function of wavelength in both the GHRS and IUE/SWP data for 10 Lac. Figure 12 depicts (small crosses) the differences between the laboratory lines referred to in the Brandt et al. (1998) study and the measurements they made from their atlas lines (these differences have been corrected for the radial velocity of the star). We also plot as a solid line the cross-correlation results between the GHRS atlas and ensemble of SWP IUE spectra at each $S W P$-order bin (cf. Fig. 9). This line shows mean fluctuations of $\pm 3 \mathrm{~km} \mathrm{~s}^{-1}$. Its excursions from zero are always less than $\pm 3 \mathrm{~km} \mathrm{~s}^{-1}$. From these results we conclude that there are no significant differences between the wavelengths of the GHRS and SWP camera. To some degree, this is not unexpected because both calibrations were made using the same line list. Even so, the calibrations were derived using different algorithms. In particular, the GHRS wavelength solutions were obtained by a block-grouping of lines in the entire spectral domain (Lindler 1993) whereas the $I U E$ solutions were performed for individual echelle orders for wavelengths greater than $\lambda 1350$.

Figure 12 shows no trend in the mean velocities for echelle orders grouped above and 
below the $\lambda 1350$ demarcation. We can expect that both random and systematic errors of the more current Pt wavelengths are smaller than the older, heterogeneous sources. For example, one may attribute the increased scatter of points in this figure below $\lambda 1500$ to random errors from the older laboratory studies. Similarly, any systematic errors in the older laboratory data would show up as trends away from both the zero-level and the solid line. The dashed line in the plot shows evidence of possible systematics in the laboratory data in the wavelength range $\lambda \lambda 1650-1750$ and $\lambda \lambda 1190-1250$. Detailed inspection of the line list from which the GHRS wavelengths are drawn suggests that such descrepancies are not limited to one type of ion. For example, several ions of both light and Fe-like ions are represented in these wavelength regions.

\subsection{Comparison with Other Studies}

Two papers (González-Riestra et al. 2000, Holberg, Barstow, \& Sion 1998) have reported that $N E W S I P S$-processed, high-dispersion $S W P$ images have negative zero-points errors relative to other standards. Both rely upon measurements of centroids of individual interstellar lines, generally in spectra of OB stars. González-Riestra et al. reported differences of $-17.7 \pm 3.7 \mathrm{~km} \mathrm{~s}^{-1}$ for the $S W P$ camera with respect to ground-based measurements of interstellar lines in the optical spectra of the same stars. A smaller discrepancy was found for small-aperture, long-wavelength camera data. As a consequence, a correction of $+17.7 \mathrm{~km} \mathrm{~s}^{-1}$ was applied to all $S W P$ high-dispersion spectra in the IUE Newly Extracted Spectra ("INES") system (González-Riestra et al. 2000).

Upon investigation we are unable to support the conclusion by González-Riestra et

al. that a significant difference in radial velocities exists for NEWSIPS SWP spectra. We have attempted without success to reconcile the differences between their results and ours. 
These authors kindly provided us with program data lists, so we were able to compare cross-correlation shifts from their samples with those from entire sets of obervations of these stars in the archives. A comparison of the results showed no noticeable difference between the two samples of observations, and thus one must look elsewhere to resolve the difference. Next, we cross-correlated the same interstellar lines used in the González-Riestra et al. analysis for in a sample of 145 large-aperture SWP spectra of 10 Lac with the interstellar lines in the Brandt et al. (1998) GHRS atlas. For the three sets of lines they measured (Si II $\lambda 1259-60$, O I $\lambda 1302$, Si II $\lambda 1304$, and C III $\lambda 1335-6$ ), we found a difference $\mathrm{RV}_{S W P}$ - $\mathrm{RV}_{G H R S}=-0.9 \pm 1.5 \mathrm{~km} \mathrm{~s}^{-1}$. Thus, there is essentially no difference between the systemic velocities of these interstellar lines and the photospheric lines in the IUE spectrum for these two instruments. González-Riestra et al. did not measure SWP observations of 10 Lac, but their measurements of lines in HD 93521 and HD 60753 spectra suggest that the wavelengths in these stars typify the zero-point offsets they found for their total sample of six stars: $-18.8 \mathrm{~km} \mathrm{~s}^{-1}$ for HD 93521 and $-12.9 \mathrm{~km} \mathrm{~s}^{-1}$ for HD 60753. In $\$ 4.2 .3$. we reported that the relative velocities of the photospheric lines for these three stars were in agreement with ground-based studies, so in this important respect all our comparisons are self-consistent (as are those of González-Riestra et al.).

Since we can find no other discrepancy among our results with respect to the atlas results or with respect to photospheric lines of the stars in our common sample, we are left with the possibility that the measurement positions of the interstellar lines by González-Riestra et al. are misleading for some subtle reason. One possibility is that the multi-component nature of the interstellar clouds toward these stars gives different centroid velocities for some groups of lines than for others. In particular, differing degrees of saturation among interstellar lines at low spectral resolutions can give rise to rather large centroid velocities differences among various interstellar line complexes in the spectrum of a given star. For example, differences between the $\lambda 1304$ and $\lambda 1335-6$ complexes are 
quite large in the spectrum of HD 93521, as was indeed noted by González-Riestra et al. themselves.

In $\$ 3.1 .1$. we noted a very similar time-dependence of apparent velocities between IUESIPS and NEWSIPS spectra with respect to the dependence noted by Holberg, Sion, \& Barstow (1998). These authors also found a mean difference of $-8.3 \mathrm{~km} \mathrm{~s}^{-1} \pm 1.4$ between NEWSIPS -derived and ground-based radial velocities for four white dwarfs. This finding appears to contradict the conclusion from our work on 10 Lac and other bright B stars (\$4.2.) that the velocity zero-point for SWP NEWSIPS is correct within $-1 \mathrm{~km} \mathrm{~s}^{-1}$. We have attempted to track down the source of this discrepancy in a number of ways. First, in $\$ 3.2$. we discussed the possibility that the mode of centering on bright stars and faint stars could lead to different results (see Table 1). Yet, we found no such effect. We may also consult Fig. 4 for clues of an apparent velocity difference between white dwarfs and bright OB stars. The figure implies that the mean of the white dwarf velocity offset should agree within about $\pm 1 \mathrm{~km} \mathrm{~s}^{-1}$ of our 10 Lac result. This statement holds true for the velocity average of the four white dwarfs selected by HSB. We have also cross-correlated the groups of interstellar lines considered by HSB in selected echelle orders. These comparisons produce a mean IUESIPS - NEWSIPS velocity shift of $-1.3 \pm 0.6 \mathrm{~km} \mathrm{~s}^{-1}$ relative to differences derived from orders containing only photospheric lines. Thus, there is nothing special or "peculiar" about the spatial mapping of the ISM lines in NEWSIPS that would give results systematically different from an analysis of photospheric features over the whole spectrum.

Unable to resolve a disagreement with HSB's results in this manner, we proceeded with another test. In their discussion of the apparent wavelength error, HSB compared their results with two earlier studies of interstellar lines in their white dwarf sample. The more accurate of these was a study by Lemoine et al. (1996; Small Science Aperture observations) of the ISM line spectrum of the white dwarf G191-B2B. To make a comparison 
with NEWSIPS wavelengths, we acquired the Lemoine et al. data from the the MAST archives. These observations were originally made by substepping the grating to minimize fixed pattern detector noise, so we made corrections for these shifts by comparing emission line spectra obtained at nearly the same time for wavelength calibration (this comparison was made by measuring centroid line positions of the two groups of spectra interactively). The differences we determined were applied to the raw data in order to form high-quality, co-added spectra. We next cross-correlated 13 SWP LGAP spectra of the star with the mean GHRS spectrum in narrow wavelength regions centered on 12 interstellar features studied by these Lemoine et al. These cross-correlations gave a mean shift, $\mathrm{RV}_{N E W S I P S}$ $\mathrm{RV}_{\text {GHRS }}$, of $-2.3 \pm 3.0 \mathrm{~km} \mathrm{~s}^{-1}$, i.e. zero within the errors (see Table 3). This finding is at mild variance with the HSB prediction of $-8.2 \mathrm{~km} \mathrm{~s}^{-1}$, but it is consistent with our other results.

\section{Conclusions}

Cross-correlation tools have been used to determine whether systematic trends in wavelength zero-points exist with respect to camera aperture, time, focus, and THDA for high-dispersion $I U E / N E W S I P S$ data for all three cameras. Not surprisingly, a major residual error source is in an implied inconsistency in target-centering/guiding among different observations, some of which could arise from different thermal histories of the telescope. Even so, the wavelength calibration of the $S W P$ high-dispersion data is surprisingly good. In fact, it can rival the wavelength precision of the Large Aperture GHRS spectra for stars observed many times over several epochs and in consistent instrumental

conditions. Even over 10-15 years, we found evidence of significant velocity trends for only one or two stars (3.3.). At most these trends could amount to $\pm 3-5 \mathrm{~km} \mathrm{~s}^{-1}$ over this duration, and they may actually be smaller. We have also found that dedicated time-series 
observations of bright stars can substantially reduce apparent velocity errors. Yet, even for such data strings, changes in the spacecraft environment can produce spurious variations of $\pm 3 \mathrm{~km} \mathrm{~s}^{-1}$ over a timescale of a few hours. It is not clear that series of long-wavelength spectra exist in $I U E$ database to detect such effects, but we expect that they are present nonetheless.

Using the GHRS atlas of 10 Lac as an secondary standard, we have found that the apparent radial velocity determined from high-dispersion images of the IUE SWP camera is zero, within the errors of measurement, with respect to the ultraviolet wavelength system defined by contemporary laboratory catalogs (Brandt et al. 1997). This result has been checked by lower-accuracy comparisons such as the difference between apparent radial velocities from $S W P$ echellograms of two other presumed constant early-type stars with similar spectral type, HD 93521 and HD 60753, and also by comparing wavelengths of several interstellar lines with those measured by the GHRS. Additionally, and for all three IUE cameras, we have confirmed the accuracy of the zero-points with more limited sets of lines with the GHRS and STIS atlases of Procyon and Arcturus, respectively. We have compared the zero-points of the two IUE long-wavelength cameras by means of the Arcturus and Procyon atlases and also by compared wavelengths within a common narrow wavelength order among the three cameras. Neither of these comparisons led to a siginificant radial velocity offset between the IUE and HST instruments. The long-wavelength camera results are also in agreement with the ground-based velocity of Procyon, but less precisely so. Our estimate of the accuracy of in the zero-point error for the $L W P$ and $L W R$ cameras is itself imprecise, but judging from its consistency with the Arcturus and Procyon atlas wavelengths, it is probably no worse than $\pm 5 \mathrm{~km} \mathrm{~s}^{-1}$. Our findings are in apparent disagreement with those of González-Riestra et al. (2000). Their results led to shifts in the wavelength scales of the $S W P$ high-dispersion and $L W P / L W R$ low-dispersion data for the INES (IUE Newly Extracted Spectra) data product produced by the European Space 
Agency.

We have found no noticeable wavelength-dependent differences in zero-point between IUE SWP camera data for $10 \mathrm{Lac}$ and the GHRS atlas of this star. However, note that this finding does not address possible errors within a particular order or measurements of a common feature appearing in adjacent orders, such as the Mg II h line (see Gonźalez-Riestra et al. 1998). Although we have not examined the data for this purpose, we have noticed occasional mismatches of $5-10 \mathrm{~km} \mathrm{~s}^{-1}$ from one end of an echelle order to another compared to theoretical spectral line syntheses. Such errors probably originate from an inadequacy of the calibration algorithms to determine dispersion nonlinearities accurately because only a few lines were available in individual orders of WAVECAL echellograms.

Finally, because the GHRS atlas of 10 Lac has a broad wavelength coverage and shows good correspondence in its wavelengths compared to laboratory data, we recommend the use of this atlas as a reference standard for comparison of astronomical mid-UV wavelengths with data from other missions.

The author gained valuable technical information presented herein while serving on the staff of the IUE Project at the Goddard Space Flight Center (NASA). It is our pleasure to acknowledge informative technical discussions with Dr. Catherine Imhoff, Mr. Randall Thompson, and Mrs. Karen Levay concerning questions of IUE operations practices and detailed contents of the IUE database. We also thank Dr. Rosario González-Riestra for providing us with a list of spectra used in her team's investigation of NEWSIPS data properties. We acknowledge helpful conversations with Drs. Richard Robinson, Charles Proffitt, and Mr. Don Lindler on the calibration of GHRS wavelengths, and Dr. Gill Nave for comments on the utility of various laboratory wavelength sources. We are also indebted to to Drs. Martin Snow and Brian Wood for providing their atlas data to MAST, and to Dr. Tom Ayres for providing it before publication. We are grateful to Drs. Rosario 
Gonzalez-Riestra and Jay Holberg for providing unpublished data from their work. We wish to thank Dr. Marc Postman for his patience. 


\section{REFERENCES}

Ayres, T. R., Brown, A., Harper, G. M. et al. 1999, BAAS, 194, 6701A

Barstow, M. A., Holberg, J. B., Bruhweiler, F. C., Collins, J. 1996, AJ, 111, 2361

Brandt, J. C., S. R. Heap, et al. 1998, AJ, 116, 941

Fullerton, A. W. 1990, Ph. D. Dissertation, Univ. of Toronto

Garhart, M. P., Smith, M. A., Levay, K. L, Thompson, R. W., \& Turnrose, B. E. 1997, NEWSIPS Manual, IUE NASA Newsletter No. 57

Garhart, M. P., Smith, M. A., Levay, K. L, Thompson, R. W., \& Turnrose, B. E. 1997, NEWSIPS Manual, IUE NASA Newsletter No. 57

González-Riestra, R., Cassatella, A., Solano, E., Altamore, A., \& Wamsteker, W. 2000, Astr. Ap. Suppl, 141, 343

Grayzeck, E. J., \& Kerr, F. J. 1974, AJ, 79, 368

Hobbs, L. M. 1969, ApJ, 157, 135

Holberg, J. B., Barstow, M. A., \& Sion, E. M. 1998, ApJS, 119, 207

Irwin, A. W., Fletcher, J. M., Yang, S. L. S., Walker, G. A. H., \& Goodenough, C. 1992, PASP, 104, 489

Leomoine, M., Vidal-Madjar, A., Bertin, P., Ferlet, R., Gry, C., \& Lallement, R. 1996, Astr. Ap., 308, 601L

Lindler, D. 1993, in Calibrating Hubble Space Telescope, ed. J. C. Blades \& S. J. Osmer (Baltimore: STScI), p. 278

Reader, J., Acquista, A., Sansonetti, C. J., \& Sansoneti, J. E. 1990, ApJS, 72, 831

Robinson, R. D. 2000, priv. commun. 
Rogerson, J. B., Jr., \& Upson, W. L. II 1977, ApJS, 35, 37

Stokes, G. M. 1978, ApJS, 36, 115

Walborn, N. R. 1972, AJ, 77, 312

Wilson, R. E. 1953, General Catalogue of Stellar Radial Velocities (Washington: Carnegie Inst).

Wood, B. E., Harper, G. M., Linsky, J. L., Dempsey, R. C. 1996, ApJ, 458, 761 
Table 1: Large Minus Small Aperture Zero-Point Differences $\left(\mathrm{km} \mathrm{s}^{-1}\right)$

\begin{tabular}{|c|c|c|c|c|c|c|}
\hline SWP Camera: & & & & & & \\
\hline Bright Stars: & $10 \mathrm{Lac}$ & $\tau$ Sco & $\zeta \mathrm{Oph}$ & $\eta \mathrm{UMa}$ & $\lambda$ Lep & $\zeta$ Cas \\
\hline LGAP - SMAP & 0.4 & -3.5 & -0.6 & +0.2 & +0.2 & -1.7 \\
\hline No. LGAP: & 145 & 74 & 59 & 48 & 40 & 54 \\
\hline No. SMAP: & 6 & 33 & 15 & 12 & 10 & 12 \\
\hline Faint Stars: & RR Tel & Sirius B & $\mathrm{BD}+75^{\circ} 325$ & $\mathrm{BD}+28^{\circ} 4211$ & HD 93521 & HD 60753 \\
\hline LGAP - SMAP: & -2.5 & -2.3 & -4.7 & 2.4 & 2.1 & 0.7 \\
\hline No. LGAP: & 46 & 3 & 86 & 76 & 99 & 82 \\
\hline No. SMAP: & 5 & 1 & 5 & 2 & 14 & 1 \\
\hline LWP Camera: & $\tau$ Sco & $\eta \mathrm{UMa}$ & HD 93521 & & & \\
\hline LGAP - SMAP & -2.5 & -1.1 & 0.1 & & & \\
\hline No. LGAP: & 75 & 156 & 20 & & & \\
\hline No. SMAP: & 15 & 4 & 2 & & & \\
\hline LWR Camera: & $10 \mathrm{Lac}$ & $\tau$ Sco & $\zeta \mathrm{Oph}$ & RR Tel & & \\
\hline LGAP - SMAP & 1.9 & -1.0 & -4.3 & +4.3 & & \\
\hline No. LGAP: & 3 & 24 & 11 & 15 & & \\
\hline No. SMAP: & 4 & 14 & 7 & 3 & & \\
\hline
\end{tabular}


Table 2: Linear Regression Velocity Slopes with Time for LGAP Data $\left(\mathrm{km} \mathrm{s}^{-1} \mathrm{yr}^{-1}\right)$

\begin{tabular}{|c|c|c|c|c|c|c|c|c|}
\hline $\begin{array}{r}\text { Star/Cam.: } \\
\text { SWP }\end{array}$ & $10 \mathrm{Lac}$ & $\tau$ Sco & $\zeta \mathrm{Oph}$ & $\eta \mathrm{UMa}$ & $\lambda$ Lep & $\zeta$ Cas & HD 60753 & $+75^{\circ} 325$ \\
\hline Slope: & -0.01 & -0.05 & -0.29 & -0.49 & -0.13 & -0.03 & -0.53 & -0.34 \\
\hline No. $\sigma:$ & $(<1)$ & $(<1)$ & 2.1 & $(1.5)$ & $(1.0)$ & $(<1)$ & $>4$ & 4 \\
\hline \multicolumn{9}{|l|}{ LWP } \\
\hline & $10 \mathrm{Lac}$ & $\tau$ Sco & $\eta \mathrm{UMa}$ & $+28^{\circ} 4211$ & $\zeta$ Cas & $+75^{\circ} 325$ & RR Tel & HD 93521 \\
\hline Slope: & -0.44 & -0.10 & 0.01 & 0.04 & 0.28 & -0.03 & 0.05 & .03 \\
\hline No. $\sigma$ : & $(<1)$ & $(<1)$ & $(<1)$ & $(<1)$ & $<1$ & $<1$ & $<1$ & $<1$ \\
\hline \multicolumn{9}{|l|}{ LWR } \\
\hline No. $\sigma:$ & $(<1)$ & $(<1)$ & 3.1 & $(1.5)$ & $(1.0)$ & $(<1)$ & & \\
\hline Star: & $\tau$ Sco & $\zeta \mathrm{Oph}$ & $\zeta$ Cas & $+28^{\circ} 4211$ & & & & \\
\hline Slope: & -.57 & -0.55 & 0.28 & -.28 & -0.34 & -1.7 & & \\
\hline No. $\sigma:$ & $(<1)$ & $(<1)$ & $(<1)$ & $(<1)$ & & & & \\
\hline
\end{tabular}


Table 3: Radial Velocity Zero-Point Results ("Other" - IUE $\mathrm{ISMAP}_{\text {) }}$

\begin{tabular}{|c|c|c|}
\hline Camera & Comparison Made & Mean RV Diff \\
\hline \multicolumn{3}{|l|}{$S W P$} \\
\hline & UV lab via GHRS atlas: 10 Lac (main result) & $-0.9 \pm 3.5 \mathrm{~km} \mathrm{~s}^{-1}$ \\
\hline & Procyon atlas (GHRS, em. lines) & $-3.9 \pm 5 \mathrm{~km} \mathrm{~s}^{-1}$ \\
\hline & Arcturus atlas (STIS, continuum) & $-0.7 \pm 5 \mathrm{~km} \mathrm{~s}^{-1}$ \\
\hline & Cross-corr. HD 93521 with 10 Lac (IUE) & $+1.8 \pm 3 \mathrm{~km} \mathrm{~s}^{-1}$ \\
\hline & Cross-corr. HD 60753 with 10 Lac (IUE) & $-0.5 \pm 3 \mathrm{~km} \mathrm{~s}^{-1}$ \\
\hline & Cross-corr. with GHRS of ISM lines (G191-B2B) & $-2.3 \pm 3 \mathrm{~km} \mathrm{~s}^{-1}$ \\
\hline \multicolumn{3}{|l|}{$L W R$} \\
\hline & Procyon atlas (GHRS) & $-3.5 \pm 6 \mathrm{~km} \mathrm{~s}^{-1}$ \\
\hline & Arcturus atlas (STIS) & $+2.7 \pm 3 \mathrm{~km} \mathrm{~s}^{-1}$ \\
\hline \multicolumn{3}{|l|}{$L W P$} \\
\hline & Procyon atlas (GHRS) & $+7.9 \pm 6 \mathrm{~km} \mathrm{~s}^{-1}$ \\
\hline LWR - LWP: & $\mathrm{X}$-corr. of 6 stars & $+0.4 \pm 3 \mathrm{~km} \mathrm{~s}^{-1}$ \\
\hline \multicolumn{3}{|c|}{ Long \& short- $\lambda$ cams. } \\
\hline & SWP - LWP: & $+3.3 \pm 4 \mathrm{~km} \mathrm{~s}^{-1}$ \\
\hline & SWP - LWR: & $-1.5 \pm 4 \mathrm{~km} \mathrm{~s}^{-1}$ \\
\hline
\end{tabular}


Table 4: Radial Velocity Differences between Long-Wavelength Cameras (LWR - LWP)

\begin{tabular}{ccr|ccr}
\hline \hline Star & Mean RV & Number & Star & Mean RV & Number \\
& $\begin{array}{c}\text { Difference } \\
\left(\mathrm{km} \mathrm{s}^{-1}\right)\end{array}$ & Spectra & & Difference & Spectra \\
& & & & \\
& & & & \\
& & & & \\
10 Lac & 1.0 & 3,13 & RR Tel & -3.5 & 21,14 \\
BD $+75^{\circ} 325$ & 3.4 & 6,67 & $\tau$ Sco & 3.3 & 24,75 \\
BD $+28^{\circ} 4211$ & -3.1 & 13,45 & HD 11636 & 1.3 & 12,2 \\
\hline
\end{tabular}


Fig. 1.- Cross-correlation shifts of 415 WAVECAL SWP echellograms during the lifetime of the IUE for orders at the opposite ends of the camera, centered at $1168 \AA$ and $1969 \AA$.

Fig. 2.- Velocity shifts of $S W P$ camera WAVECAL echellograms during the IUE lifetime. The solid line is a cubic polynomial fit (zero-point arbitrary) which shows the (incomplete) extent to which time dependences were removed from the WAVECAL wavelength zero points. Note that at certain epochs the difference between an local mean and the smooth curve may amount to $\approx 2 \mathrm{~km} \mathrm{~s}^{-1}$. Such residuals are not removed in the wavelength calibration of NEWSIPS.

Fig. 3.- Wavelength-integrated cross-correlation shifts of WAVECAL echellograms of the two IUE long-wavelength cameras during IUE lifetime. Shifts represent wavelength zeropoint differences and are expressed as velocities. Solid line depicts the fit with a linear $(L W P)$ and cubic $(L W R)$ function.

Fig. 4.- Cross-correlation shift differences in the sense IUESIPS - NEWSIPS with time. Displayed are shifts for echellograms observed for the velocity standards (as labeled). The white dwarfs are those studied by Holberg et al. (1998). Vertical dotted lines indicate the times in which new wavelength calibration constants were applied to the IUESIPS processing software according to Thompson et al. (1981), Thompson et al. 1983, Thompson \& Turnrose, 1983, Gass \& Thompson 1985, \& Garhart 1993).

Fig. 5.- Apparent velocity trends with time: (a) HD 60753 and (b) BD+75 325. All data were obtained through the large aperture. 
Fig. 6.- Cross-correlation shifts of velocity, telescope focus, and THDA temperature for an intensive time series of $S W P$ high-dispersion observations of $\alpha$ Eridani. Dotted and dashed lines (shown for reference only) represent the $\mathrm{x}$ - and $\mathrm{y}$-components of the satellite's orbital motion ("x" is directed toward the Sun; "y" its perpendicular in the Earth's equatorial plane). THDA is in degrees Centigrade; focus is in instrumental units.

Fig. 7.- Cross-correlation shifts of velocity, telescope focus, and THDA temperature for an intensive time series of $S W P$ high-dispersion observations of $\epsilon$ Persei. For reference only: a cross-correlation between the apparent velocities and the $\mathrm{x}$-component of the (diurnal) orbital velocity shows that the velocities show a mean lag of 0.15 cycle behind the $\mathrm{x}$-component.

Fig. 8. - Velocity differences versus IUE telescope focus for echellograms of the data depicted in Fig. 18.

Fig. 9.- Mean shifts of wavelength zero-points as a function of wavelength extracted from a large number of $I U E$ echellograms for 16 stars $(10 \mathrm{Lac}, \tau$ Sco, $\zeta$ Cas, $\zeta$ Oph, $\eta$ UMa, $\lambda$ Lep and the white dwarfs BD $+75^{\circ} 325, \mathrm{BD}+28^{\circ} 4211$, Sirius B, WD0005+511, NGC246, REJ0623-3, EG102, Wolf 1346, GD 394, Gl191-B2B). These shifts were obtained by crosscorrelating wavelengths in each high-dipersion echelle order for a number of test images of a star against a reference echellogram. Belts of $\pm 1 \sigma$ around the mean shifts are drawn from the distribution of the shifts for the individual stars.

Fig. 10.- Large-aperture - Small-aperture $S W P$ apparent radial velocities of 10 Lac during the lifetime of the IUE. The sample comprises 145 LGAP images and is referred to the average of 6 SMAP images. The large scatter among velocities at late epochs is likely due to acquisition of the star at a different position in the FES. This diagram shows that the instrumental $S W P$ velocities of 10 Lac exhibit no significant trend with time. Error bars are taken from Fig. 11. 
Fig. 11.- Velocity histogram for radial velocities for 10 Lac taken from Figure 10. This distribution is essentially bimodal, reflecting two sets of target centerings for late epoch observations.

Fig. 12.- Differences of GHRS - IUE (jagged line) and GHRS - laboratory (points) wavelengths for photospheric lines of $10 \mathrm{Lac}$ as a function of wavelength. The GHRS - IUE differences refer to cross-correlation shifts between lines in the 10 Lac atlas and in a group of 145 high-dispersion SWP echellograms. The GHRS - Lab differences have been shifted by $+16 \mathrm{~km} \mathrm{~s}^{-1}$ to account for the combined radial velocity of the star and an instrumental error of the GHRS relative to the laboratory system. Error bars in the lower right are for the jagged line and refer to the mean difference between adjacent $S W P$ echelle orders. 


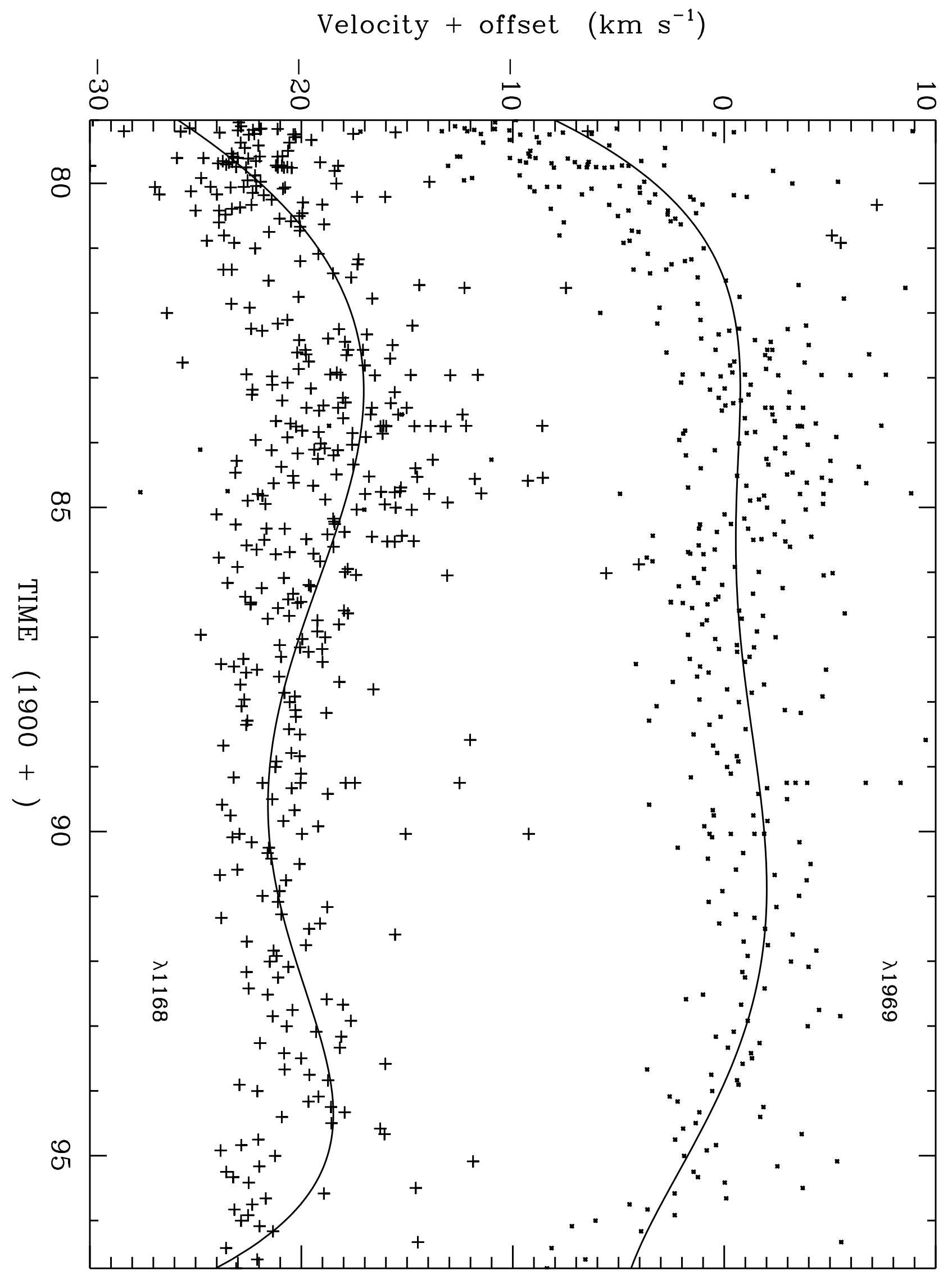




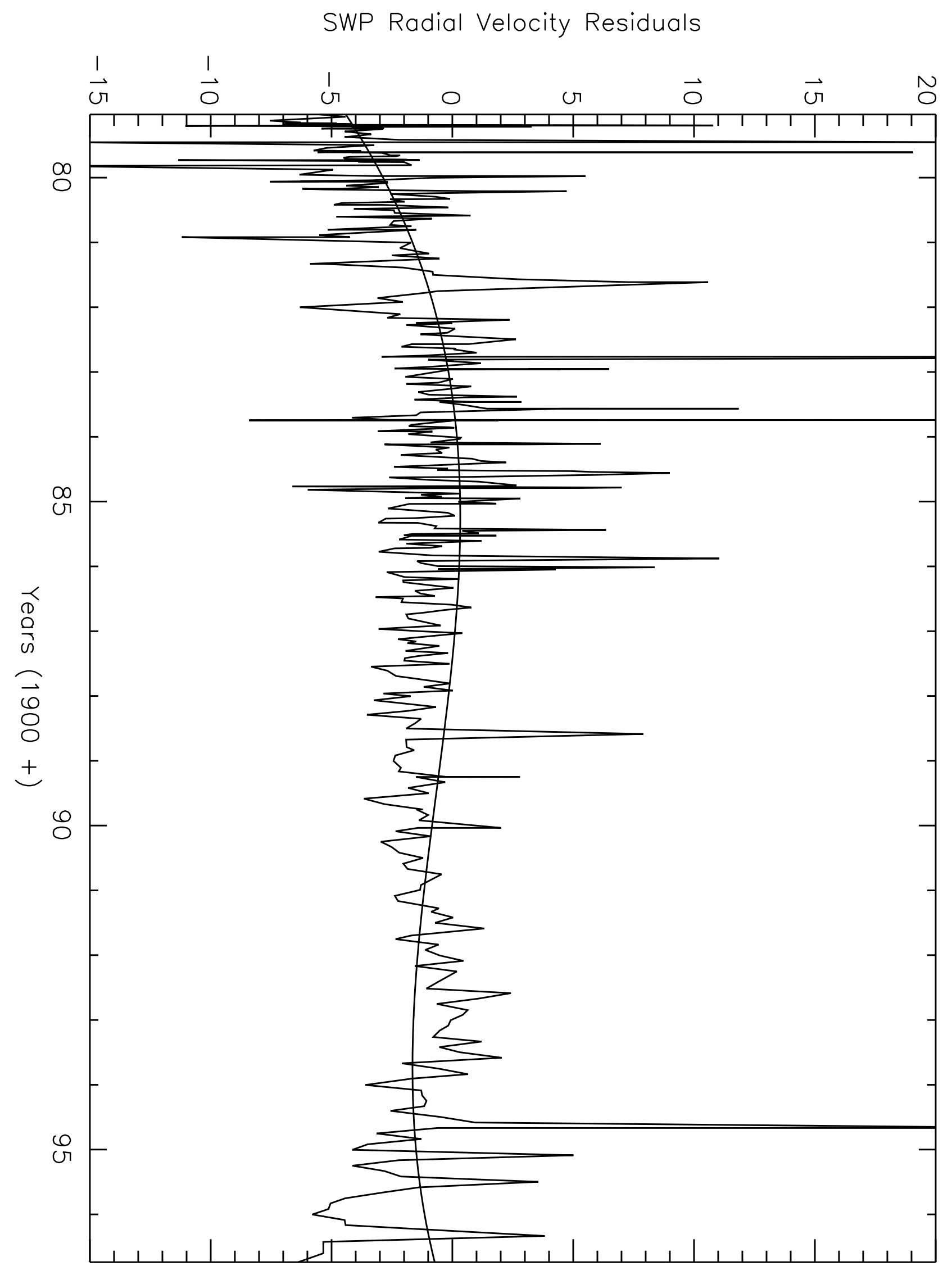




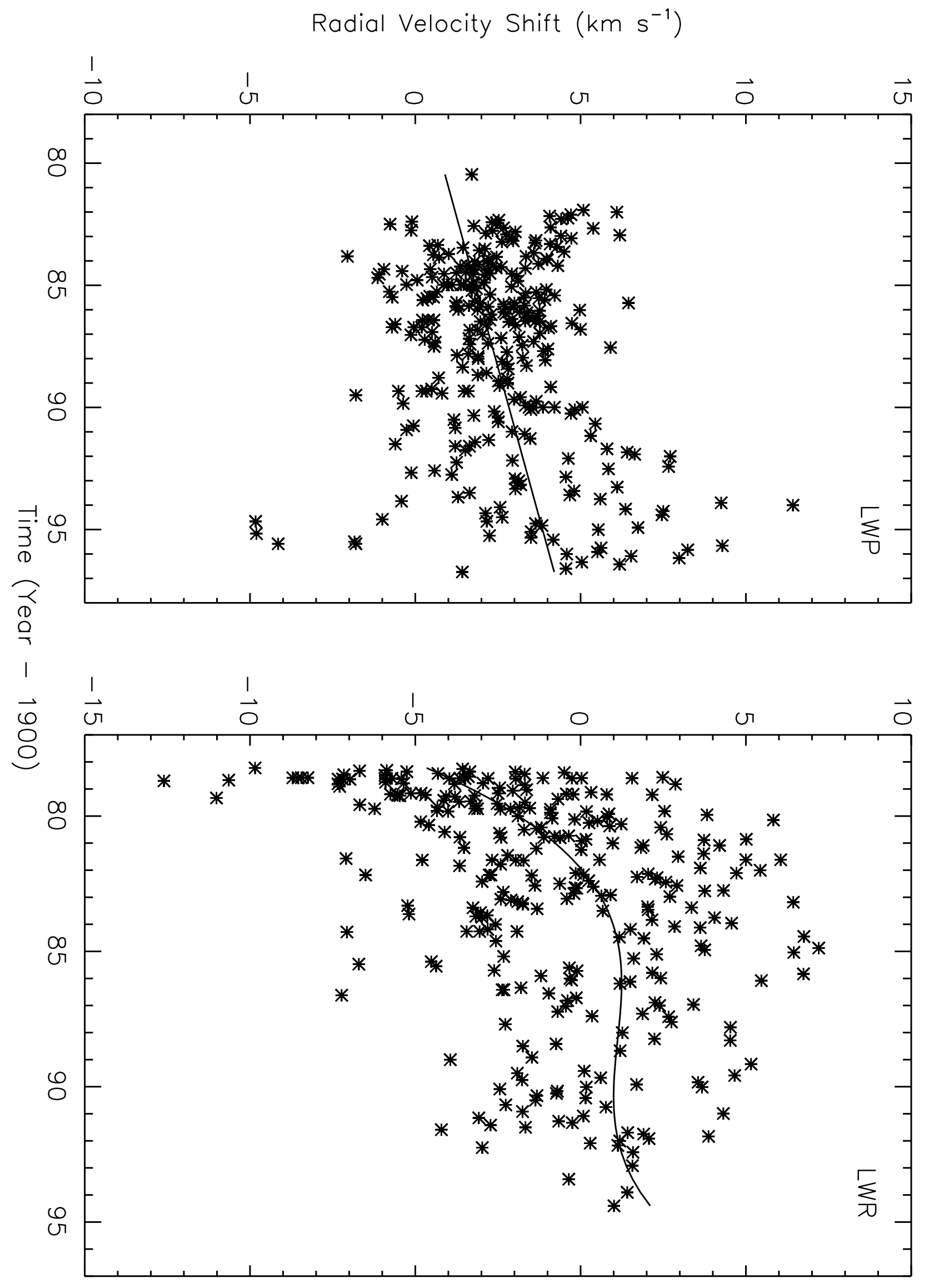




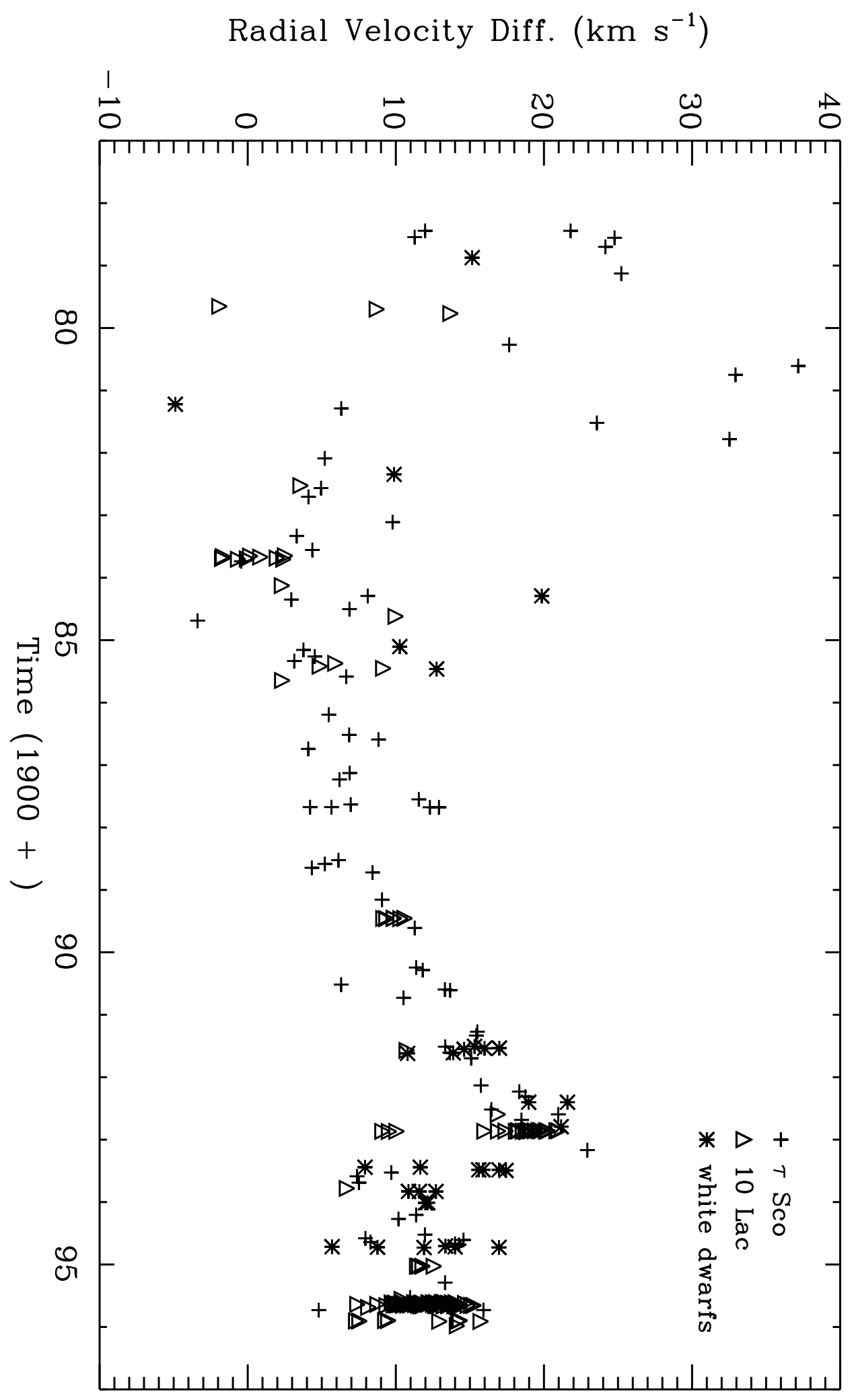




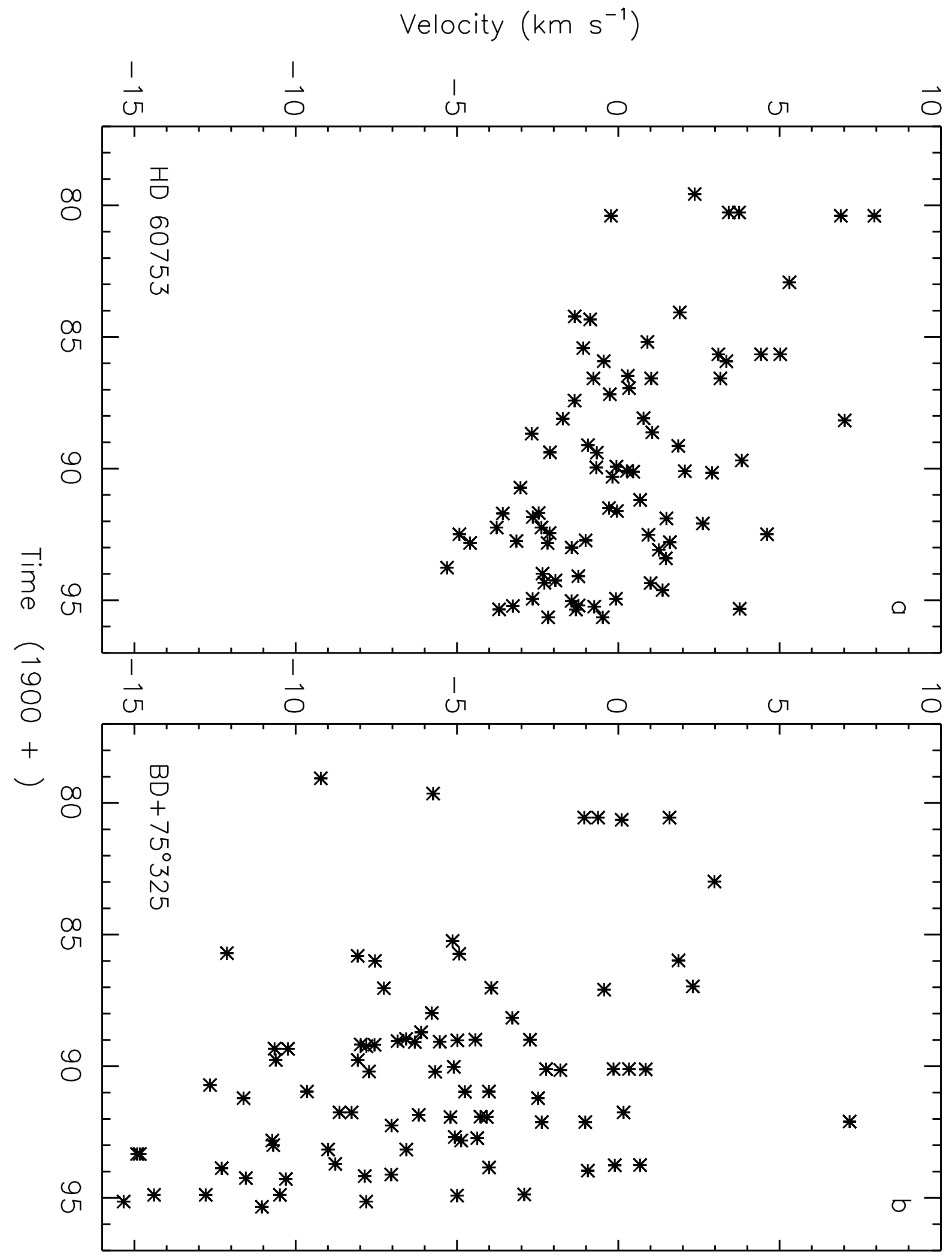




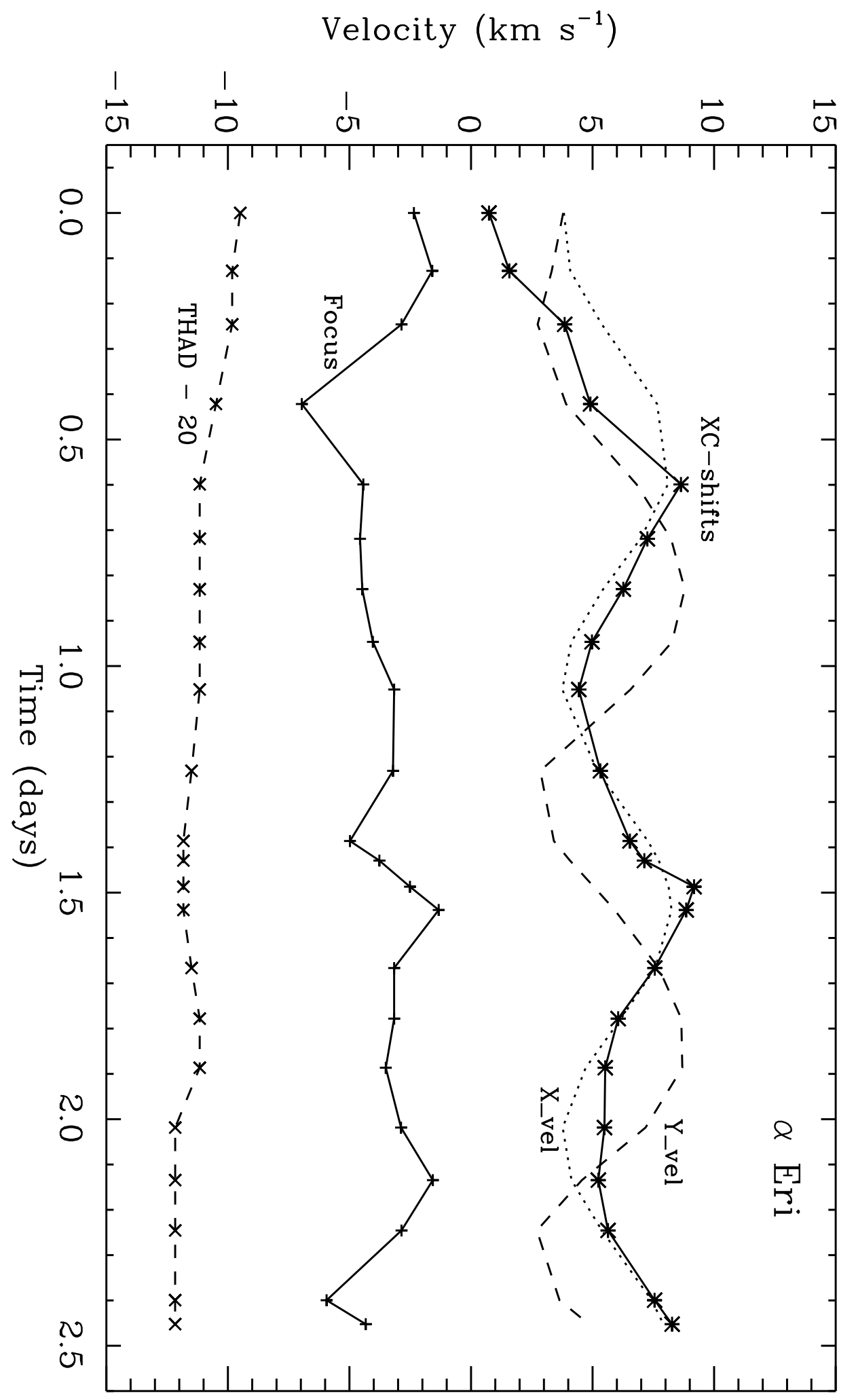




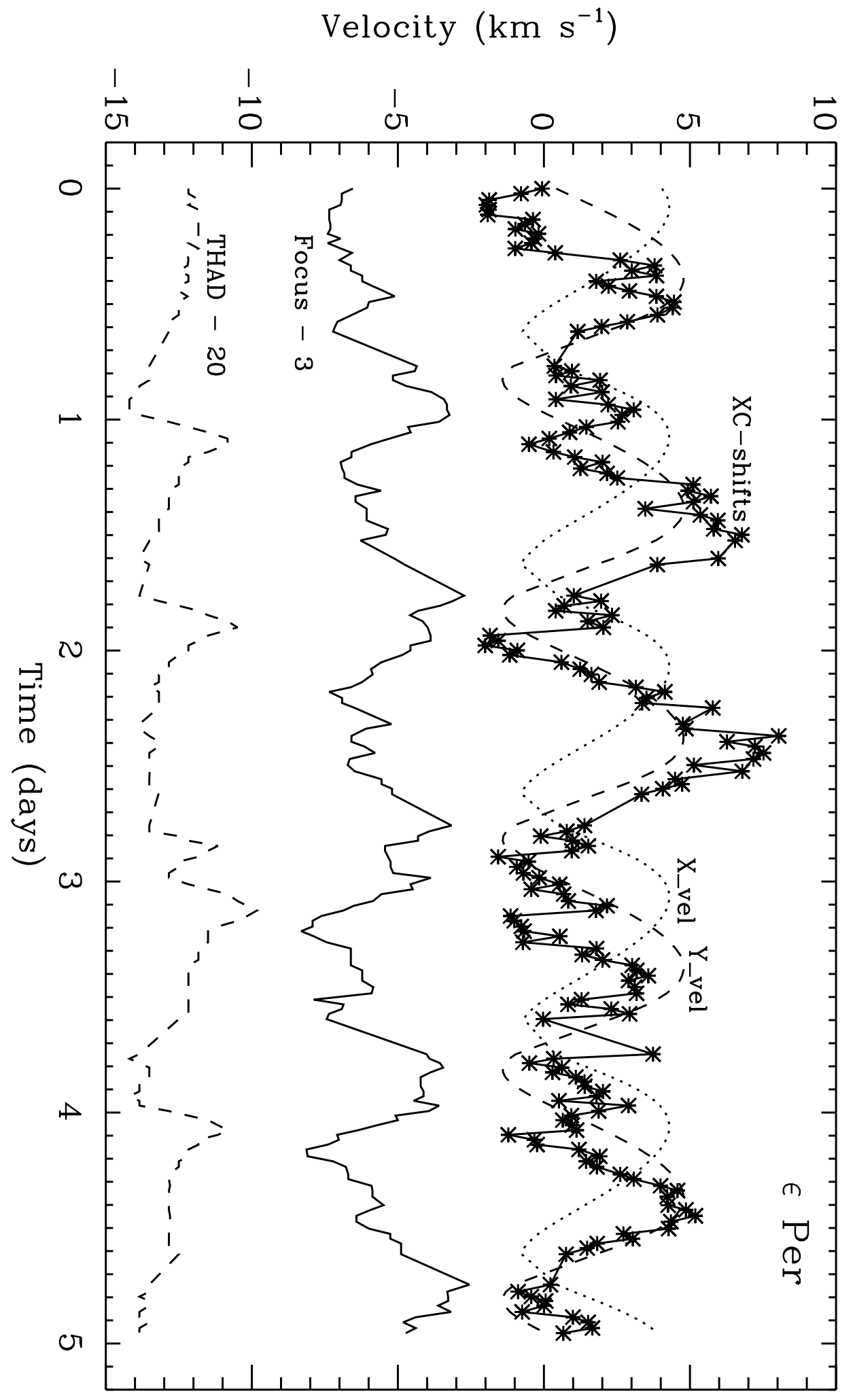




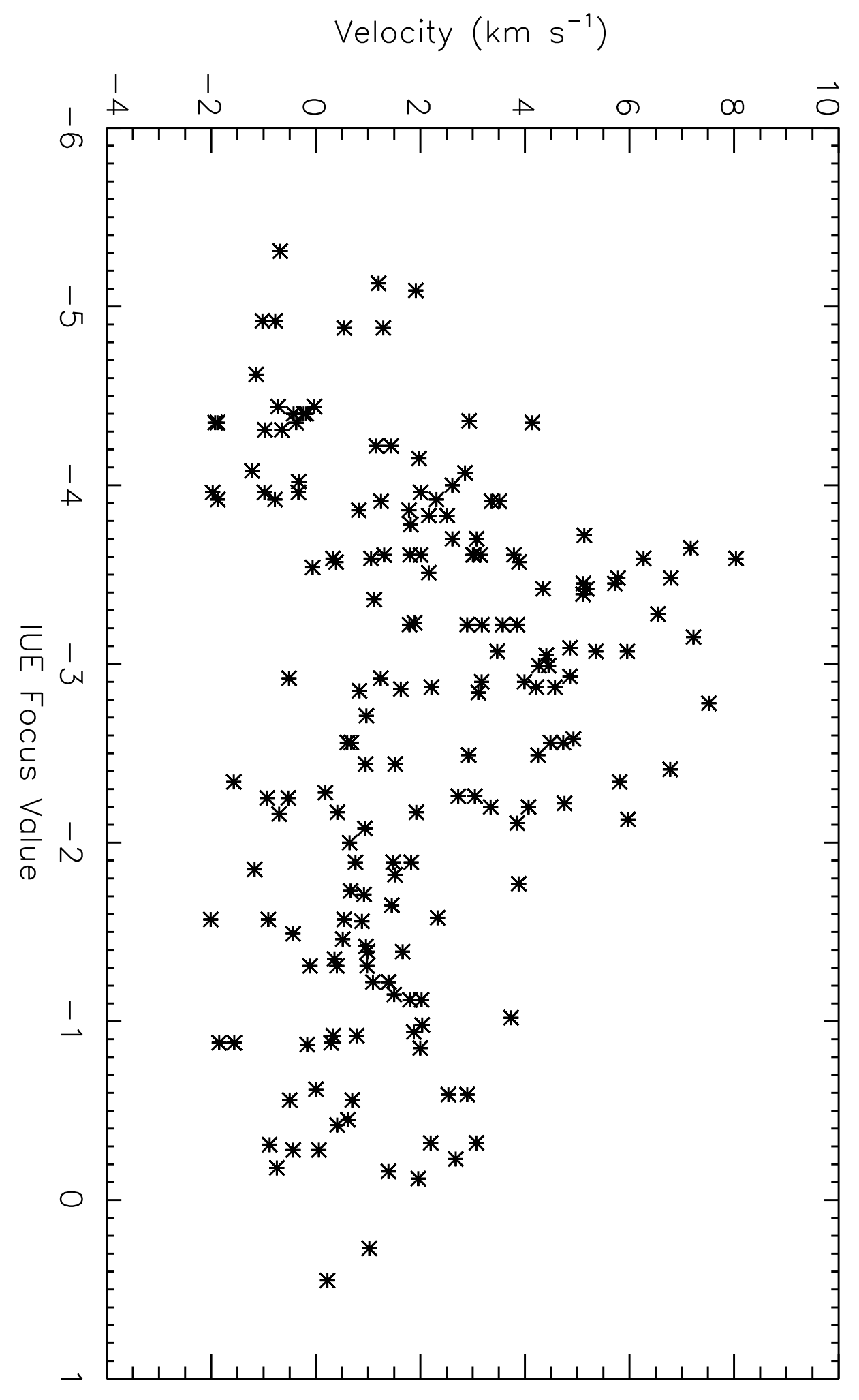




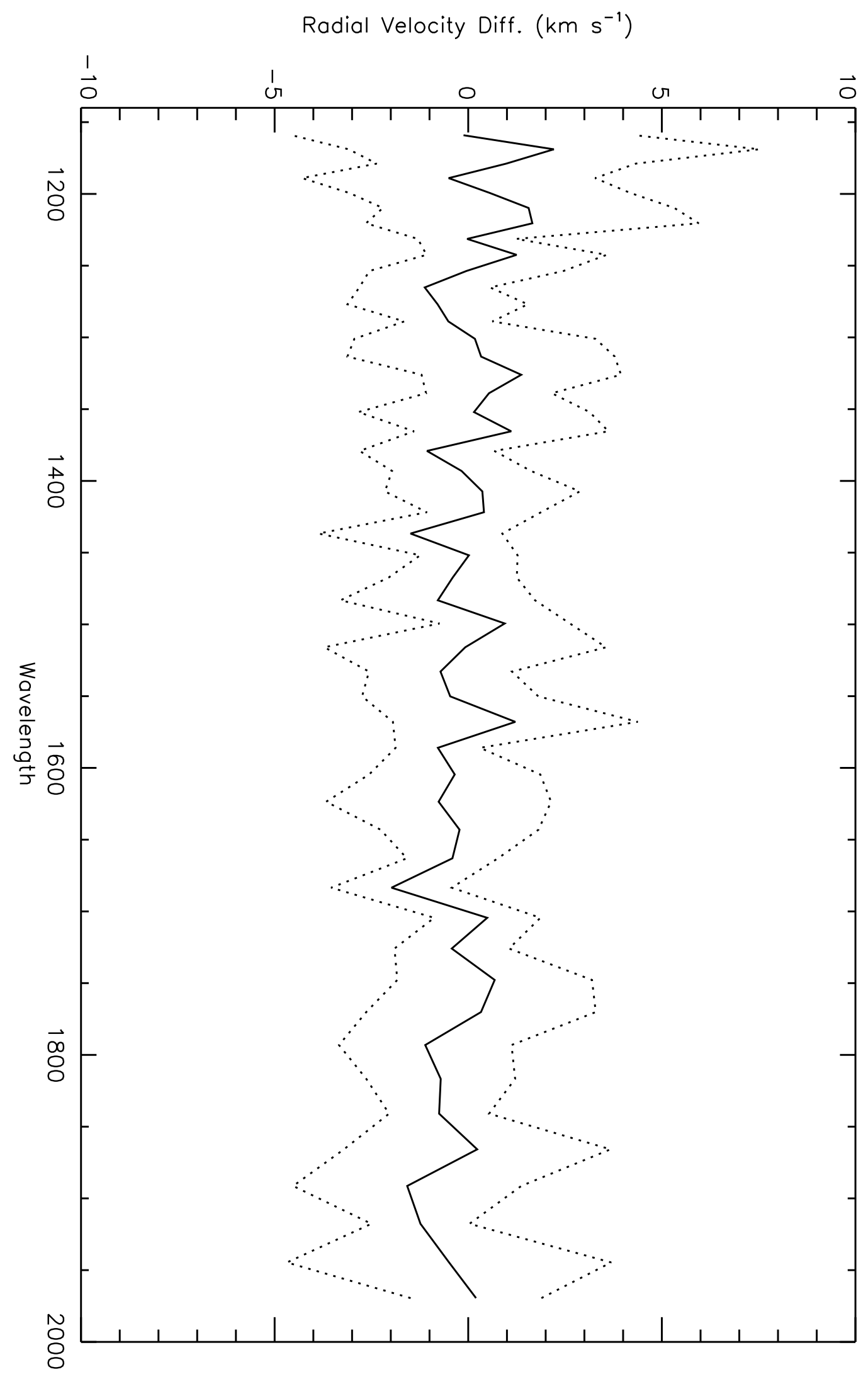




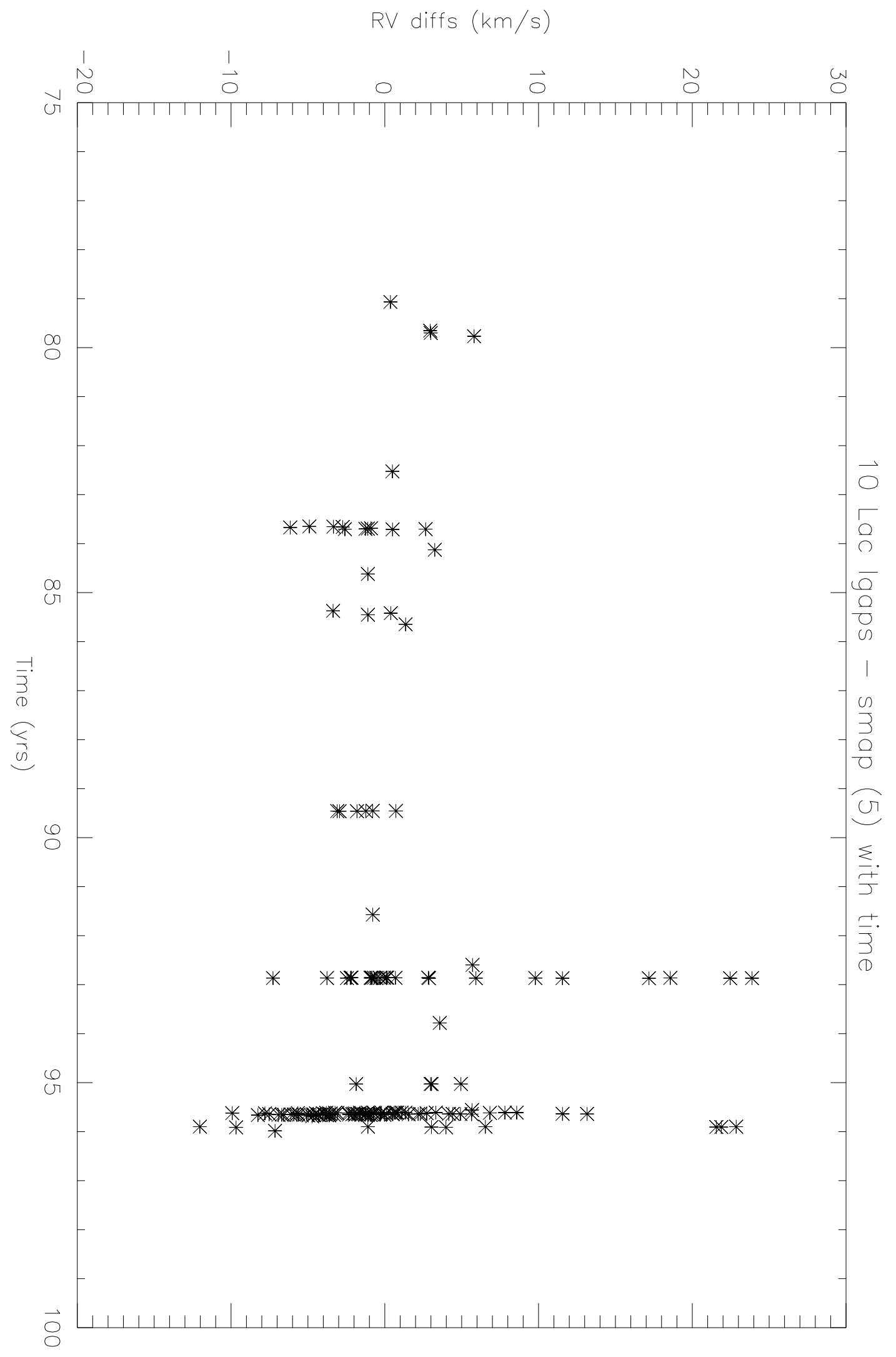




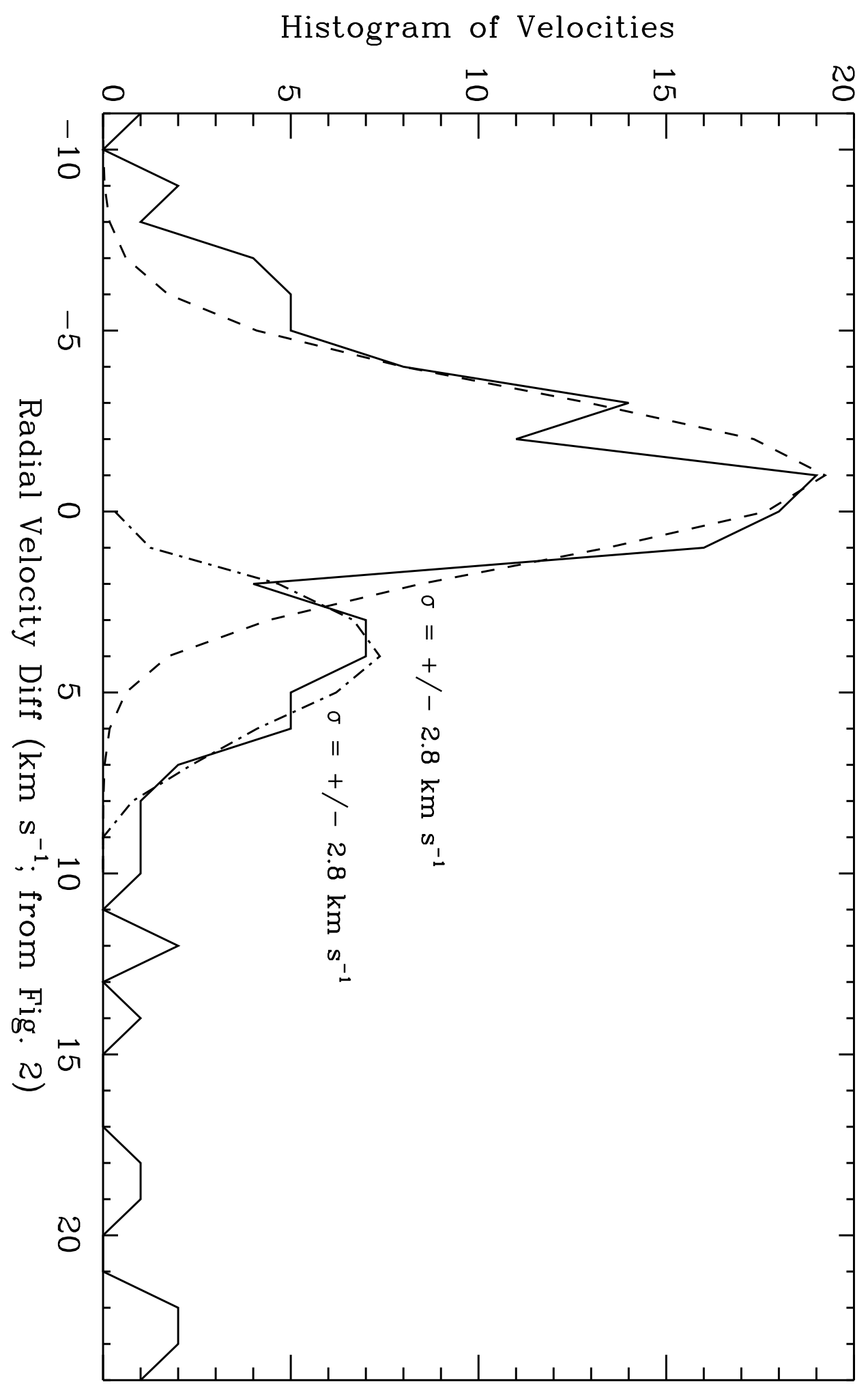




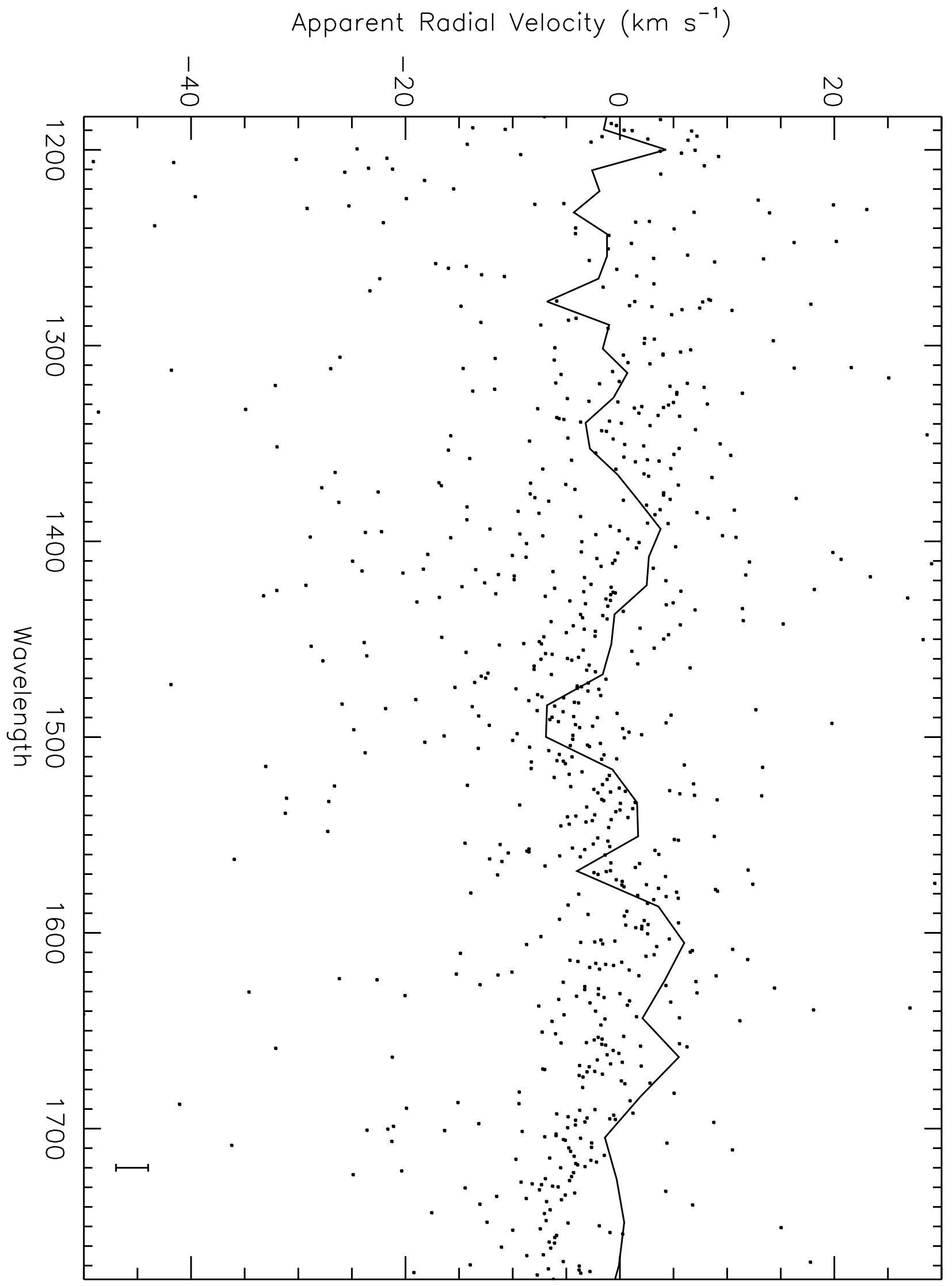

